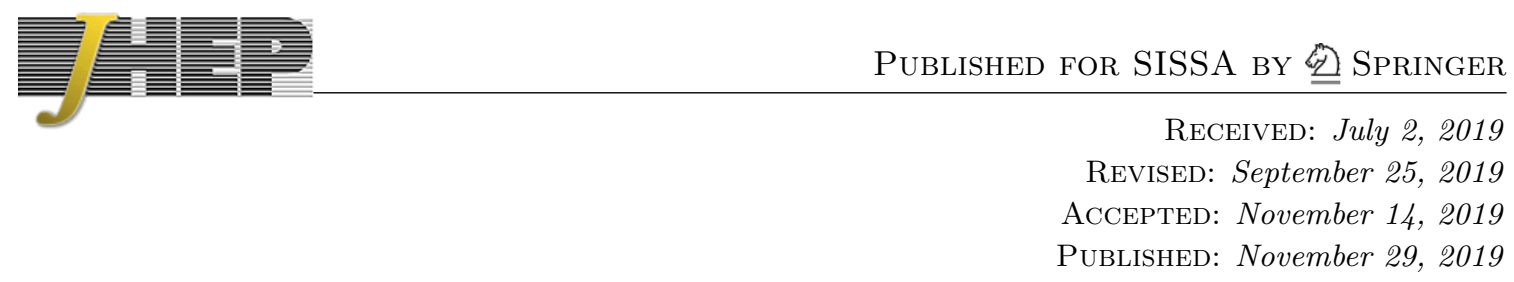

\title{
Fitting the strong coupling constant with soft-drop thrust
}

\author{
Simone Marzani, ${ }^{a}$ Daniel Reichelt, ${ }^{b, c}$ Steffen Schumann, ${ }^{b}$ Gregory Soyez ${ }^{d}$ \\ and Vincent Theeuwes ${ }^{b, d}$ \\ ${ }^{a}$ Dipartimento di Fisica, Università di Genova and INFN - Sezione di Genova, \\ Via Dodecaneso 33, 16146, Genoa, Italy \\ ${ }^{b}$ Institut für Theoretische Physik, Georg-August-Universität Göttingen, \\ Friedrich-Hund-Platz 1, 37077 Göttingen, Germany \\ ${ }^{c}$ Fermi National Accelerator Laboratory, \\ Batavia, IL, 60510-0500, U.S.A. \\ ${ }^{d}$ Institut de Physique Théorique, Paris Saclay University, CEA, \\ CNRS, F-91191 Gif-sur-Yvette, France \\ E-mail: simone.marzani@ge.infn.it, daniel.reichelt@uni-goettingen.de, \\ steffen.schumann@phys. uni-goettingen.de, gregory.soyez@ipht.fr, \\ vincent. theeuwes@uni-goettingen.de
}

ABSTRACT: Soft drop has been shown to reduce hadronisation effects at $e^{+} e^{-}$colliders for the thrust event shape. In this context, we perform fits of the strong coupling constant for the soft-drop thrust distribution at NLO+NLL accuracy to pseudo data generated by the Sherpa event generator. In particular, we focus on the impact of hadronisation corrections, which we estimate both with an analytical model and a Monte-Carlo based one, on the fitted value of $\alpha_{s}\left(m_{Z}\right)$. We find that grooming can reduce the size of the shift in the fitted value of $\alpha_{s}$ due to hadronisation. In addition, we also explore the possibility of extending the fitting range down to significantly lower values of (one minus) thrust. Here, soft drop is shown to play a crucial role, allowing us to maintain good fit qualities and stable values of the fitted strong coupling. The results of these studies show that soft-drop thrust is a promising candidate for fitting $\alpha_{s}$ at $e^{+} e^{-}$colliders with reduced impact of hadronisation effects.

KEYwords: Jets, QCD Phenomenology

ARXIV EPRINT: 1906.10504 


\section{Contents}

1 Introduction 1

2 Soft-drop thrust 3

$\begin{array}{lll}3 & \text { Monte-Carlo based pseudo data } & 7\end{array}$

4 Hadronisation corrections $\quad 11$

4.1 Monte-Carlo hadronisation model 11

4.2 Analytical hadronisation model 12

5 Fitting procedure $\quad 14$

6 Results 16

$\begin{array}{lll}7 \text { Conclusion } & 21\end{array}$

$\begin{array}{ll}\text { A Collinear safety } & 24\end{array}$

B Changes to the analytical calculation $\quad 25$

B.1 Resummation equations 26

B.2 Treatment of the transition point 27

B.3 End-point correction 29

$\begin{array}{lll}\text { C An analytic model for hadronisation corrections } & 30\end{array}$

C.1 Non-perturbative effects on plain thrust 30

C.2 Non-perturbative effects on soft-drop thrust 31

C.3 Shift in energy 33

D Validation of Hadronisation corrections from Monte Carlo generators 34

E Alternative treatment of the resummation uncertainty 36

\section{Introduction}

The CERN Large Hadron Collider (LHC) has recently finished its second physics run and it has entered a long shutdown phase, which is devoted to upgrades of the main experiments. Meanwhile, physics analyses employing the full dataset are being carried out, leading to results with astonishing experimental precision. The absence of any clear indications of new particles or interactions necessitates more and more detailed comparisons of experimental measurements with theoretical predictions. Correspondingly, high-accuracy 
calculations are of crucial importance in order to match the theoretical uncertainty to the ever decreasing experimental one and to gain sensitivity even to tiny deviations from the Standard Model. In this context, the theory community has put huge efforts in improving perturbative calculations in QCD, both at fixed-order and at the resummed level. This includes the development of sophisticated - and highly automated - simulation tools that turn high-precision calculations into explicit predictions for the complex final states produced in LHC proton-proton collisions.

A crucial quantity entering all these computations is the strong coupling constant $\alpha_{s}$, which needs to be known at high precision. To give an example, even the leading-order contribution to Higgs-boson production in gluon fusion at the LHC only starts at $\mathcal{O}\left(\alpha_{s}^{2}\right)$. The current value of the coupling constant as determined by the Particle Data Group is $\alpha_{s}\left(m_{Z}\right)=0.1181 \pm 0.0011$ [1], determined as the average of various different $\alpha_{s}$ extractions. This average is dominated by precise fits from lattice QCD [2-7], followed by measurements of event-shape variables at $e^{+} e^{-}$colliders [8-16].

The most accurate $\alpha_{s}$ determination from event shapes has been obtained by fitting the thrust distribution [17] to a theoretical calculation of outstanding precision, namely next-to-next-to-leading order matched to a resummed calculation which accounts for the first three towers of logarithmic contributions, i.e. NNLO $+\mathrm{N}^{3} \mathrm{LL}$ accuracy [14, 18]. Noticeably, this result $\left(\alpha_{s}\left(m_{Z}\right)=0.1135 \pm 0.0011\right)$ exhibits some tension with the world average. An analogous analysis performed for the C-parameter [16] resulted in a similar deviation. A common feature of these extractions is a sizeable non-perturbative contamination originating from the observed final states being composed of hadrons, rather than partons. The leading contribution to this component can be modelled analytically in terms of a single non-perturbative parameter $\Omega$, which is fitted jointly with the strong coupling. Unfortunately, $\alpha_{s}$ and $\Omega$ are highly correlated. It would thus be beneficial to break this degeneracy in order to ensure that the extraction of $\alpha_{s}$ is less dependent on non-perturbative physics.

In recent studies $[19,20]$, some of us put forward the idea of exploiting techniques developed in jet physics to improve on the determination of the strong coupling. Jet substructure techniques are primarily developed to search for boosted massive particles decaying hadronically. However, extensive literature on the topic (see for instance refs. [21-23] for recent reviews) has demonstrated their ability to extend the range of applicability of perturbative methods in jet physics. This essentially originates from a reduced sensitivity to very soft emissions, resulting in milder hadronisation corrections. The soft-drop algorithm [24] is particularly well-suited for this task and in ref. [19] soft-dropped versions of thrust, and related event shapes, where discussed in detail. In this paper we continue the analysis of soft-drop thrust and, in particular, focus on the effects that soft drop has on the impact of non-perturbative corrections in the extraction of $\alpha_{s}$. Furthermore, we investigate how the fit value and quality change as a function of the observable range considered for the extraction. In the absence of experimental measurements of soft-drop thrust, we perform our analysis on pseudo-data simulated with the Sherpa event generator [25, 26]. We will illustrate the reduced sensitivity of the soft-drop thrust observable to non-perturbative contributions. To this end we consider hadronisation corrections from Monte Carlo simulations as well as an analytical model. This qualifies soft-drop thrust, and jet substructure observables in general, as attractive candidates for future extractions of $\alpha_{s}$. 
Our paper is organised as follows, in section 2 we present the soft-drop thrust observable and some detail on its evaluation to NLO+NLL accuracy. In section 3 we discuss the generation of the pseudo data for the $\alpha_{s}$ fits. Our methods to account for hadronisation corrections are presented in section 4 . The fit procedure and our treatment of statistical and systematic uncertainties get illustrated in section 5. The corresponding results obtained are discussed in section 6. We draw conclusions and give a brief outlook in section 7 .

\section{Soft-drop thrust}

Soft drop [24] is an example of so-called grooming techniques. These are designed in the context of jet substructure with the aim to clean up jets from soft wide-angle radiation. The soft-drop algorithm recursively de-clusters the angular-ordered tree of a Cambridge-Aachen jet $[27,28]$ of original radius $R$ until a hard splitting is found. The $e^{+} e^{-}$version of soft drop uses hemisphere jets clustered using the $e^{+} e^{-}$version of the Cambridge-Aachen algorithm.

For each (hemisphere) jet the method proceeds as follows:

1. undo the last step in clustering thereby splitting the jet into two subjets $i$ and $j$;

2. check if the subjets pass the soft-drop condition:

$$
\frac{\min \left[E_{i}, E_{j}\right]}{E_{i}+E_{j}}>z_{\text {cut }}\left(1-\cos \theta_{i j}\right)^{\beta / 2},
$$

where $E_{i / j}$ denote the energies of the subjets, $\theta_{i j}$ is the angle between them and $z_{\text {cut }}$ and $\beta$ are parameters of the soft-drop algorithm;

3. if the splitting fails the soft-drop condition, the softer subjet is discarded (groomed away) and the steps are repeated for the resulting jet (the harder subjet);

4. if instead the subjets pass the condition, the procedure is terminated and the resulting jet is the combination of subjets $i$ and $j$.

The soft-drop algorithm features two parameters: $z_{\text {cut }}$ and $\beta$. The first determines how stringent the cut on the subjet energies is, whereas the latter provides an angular suppression to grooming. While $\beta \rightarrow \infty$ corresponds to no grooming, for $\beta=0$ no angular dependence is taken into account and the soft-drop algorithm reduces to the modified Mass-Drop Tagger (mMDT) [29, 30]. For practical purpose, we have implemented the above procedure using FastJet [31] for the jet clustering and additional manipulations.

The event shape thrust [17] is defined as

$$
T \equiv \max _{\vec{n}}\left(\frac{\sum_{i \in \mathcal{E}}\left|\vec{n} \cdot \vec{p}_{i}\right|}{\sum_{i \in \mathcal{E}}\left|\vec{p}_{i}\right|}\right),
$$

where $\vec{p}_{i}$ labels the three-momentum of particle $i$ and the sum extends over all particles in the event $\mathcal{E}$. The resulting vector $\vec{n}$ defines the thrust axis. Often the related variable

$$
\tau \equiv 1-T=\min _{\vec{n}}\left(1-\frac{\sum_{i \in \mathcal{E}}\left|\vec{n} \cdot \vec{p}_{i}\right|}{\sum_{i \in \mathcal{E}}\left|\vec{p}_{i}\right|}\right)
$$

is used instead. Henceforth, in the following we will refer to $\tau$ as thrust. 
The soft-drop thrust shape is defined following the procedure given in [19]:

1. determine the thrust axis for the full final state, i.e. without any grooming;

2. split the event into two hemispheres based on the thrust axis;

3. apply the soft-drop procedure on each hemisphere;

4. compute the thrust value for each groomed hemisphere separately, using the groomed hemisphere-jet momenta as reference axes.

The resulting value for soft-drop thrust is given by:

$$
\tau_{\mathrm{SD}}=\frac{\sum_{i \in \mathcal{E}_{\mathrm{SD}}}\left|\vec{p}_{i}\right|}{\sum_{i \in \mathcal{E}}\left|\vec{p}_{i}\right|}\left[1-\frac{\sum_{i \in \mathcal{H}_{\mathrm{SD}}^{L}}\left|\vec{n}_{L} \cdot \vec{p}_{i}\right|+\sum_{i \in \mathcal{H}_{\mathrm{SD}}^{R}}\left|\vec{n}_{R} \cdot \vec{p}_{i}\right|}{\sum_{i \in \mathcal{E}_{\mathrm{SD}}}\left|\vec{p}_{i}\right|}\right],
$$

where $\vec{n}_{L / R}$ denotes the axis for the left and right hemisphere, respectively, and the sums extend over all particles in the full event $(\mathcal{E})$, the soft-dropped event $\left(\mathcal{E}_{\mathrm{SD}}\right)$ or the hemispheres $\left(\mathcal{H}_{L / R}\right)$.

An important difference between this definition and what was presented in the first version of ref. [19] is the rescaling factor in front of the observable. This additional factor ensures collinear safety for the case $\beta=0$. The issue occurs for an event with multiple particles in one hemisphere and a single particle in the other one. While a virtual correction will not alter the observable value, a collinear real emission off the lone particle, that is soft enough to be groomed away, might alter the value of $\tau_{\mathrm{SD}}$ if the factor is not taken into account. If there are multiple particles present in the second hemisphere the collinear emission is protected from grooming by the clustering history. Furthermore, if $\beta>0$ the collinear emission will not be groomed away due to the angular suppression given in the soft-drop condition. Further details on this issue are given in appendix A.

NLO+NLL resummed predictions. In ref. [19] the resummation of soft-drop thrust at NLL accuracy has been presented. The calculation is based on the factorisation of the differential distribution in hard, soft and collinear pieces, derived using Soft Collinear Effective Theory [32]. In the limit $\tau \ll z_{\text {cut }} \ll 1$ the differential cross section can be written as

$$
\frac{d \sigma}{d \tau_{\mathrm{SD}}}=H(Q) S_{G}\left(z_{\text {cut }}, \beta\right)\left[S_{C}\left(\tau_{\mathrm{SD}}, z_{\text {cut }}, \beta\right) \otimes J\left(\tau_{\mathrm{SD}}\right)\right]^{2} .
$$

Here $H$ denotes the hard function, depending on the energy scale $Q$ only, $S_{G}$ is a global soft function accounting for soft wide-angle emissions, $S_{C}$ describes soft-collinear emissions, and $J$ denotes the jet function encapsulating the effect of hard-collinear radiation. Some detail on the various components and in particular their one-loop, i.e. NLL expressions are collected in appendix B.1. In [19] the resummed predictions were matched to the full NLO QCD result. NNLO QCD results for the original version of the observable definition have been presented in [33]. For our study here we have adjusted the calculation from [19] to the collinear-safe observable definition, resulting in an NLO+NLL accuracy of our predictions.

For the resummation contribution the approximation $z_{\text {cut }} \ll 1$ is used and no finite $z_{\text {cut }}$ corrections are included. Finite $z_{\text {cut }}$ effects are power corrections in $\tau$ for $\beta>0$, 
however for $\beta=0$ these are only power corrections in $z_{\text {cut }}$ (contributing at the leadinglogarithmic accuracy in $\tau$ ). Despite this fact we will still study values of $z_{\text {cut }}$ as high as 0.33. Here the finite $z_{\text {cut }}$ effects will only be taken into account through means of matching to fixed order. ${ }^{1}$ Fixed-order corrections are computed with the publicly available program EVENT2 $[35,36]$. To further validate the resummed calculation, we evaluated the soft-drop thrust observable in the CAESAR formalism [37], using an independent implementation in the Sherpa framework [38]. In addition a separate calculation was performed, with slightly different treatment of the resummation uncertainty. This is further detailed in appendix E.

To match the resummed result to the exact NLO QCD matrix element, i.e. the threeparton process at NLO accuracy, we consider two different matching schemes: multiplicative and $\log R$ matching [39]. In both cases we consider the cumulative distribution

$$
\Sigma\left(\tau_{\mathrm{SD}}\right)=\frac{1}{\sigma_{0}} \int_{0}^{\tau_{\mathrm{SD}}} d \tau_{\mathrm{SD}}^{\prime} \frac{d \sigma}{d \tau_{\mathrm{SD}}^{\prime}} .
$$

Multiplicative matching is defined by

$$
\Sigma\left(\tau_{\mathrm{SD}}\right)=\left(\frac{C \Sigma_{\mathrm{FO}}}{\Sigma_{\exp }}\right)_{\mathrm{FO}} \frac{\Sigma_{\mathrm{res}}}{C},
$$

where $\Sigma_{\mathrm{FO}}$ denotes the fixed-order cumulant distribution, $\Sigma_{\exp }$ the power-series expansion of the resummed cumulant $\Sigma_{\text {res }}$, and $C$ corresponds to $\Sigma_{\text {res }}$ and $\Sigma_{\exp }$ evaluated at the kinematic end-point $\tau_{\max }$. Aiming for NLO+NLL accuracy, $C=1$ holds and we need to expand the fixed-order ratio to $\mathcal{O}\left(\alpha_{s}^{2}\right)$

$$
\Sigma\left(\tau_{\mathrm{SD}}\right)=\left[1+\frac{\alpha_{s}}{\pi}\left(\Sigma_{\mathrm{FO}}^{(1)}-\Sigma_{\exp }^{(1)}\right)+\frac{\alpha_{s}^{2}}{\pi^{2}}\left(\Sigma_{\mathrm{FO}}^{(2)}-\Sigma_{\exp }^{(2)}+\Sigma_{\exp }^{(1)}\left\{\Sigma_{\exp }^{(1)}-\Sigma_{\mathrm{FO}}^{(1)}\right\}\right)\right] \Sigma_{\text {res }} .
$$

As an alternative scheme $\log \mathrm{R}$ matching is used

$$
\begin{aligned}
\log \Sigma\left(\tau_{\mathrm{SD}}\right)= & \left(\log \left[\frac{C \Sigma_{\mathrm{FO}}}{\Sigma_{\exp }}\right]\right)_{\mathrm{FO}}+\log \left[\frac{\Sigma_{\mathrm{res}}}{C}\right] \\
= & {\left[\frac{\alpha_{s}}{\pi}\left(\Sigma_{\mathrm{FO}}^{(1)}-\Sigma_{\exp }^{(1)}\right)+\frac{\alpha_{s}^{2}}{\pi^{2}}\left(\Sigma_{\mathrm{FO}}^{(2)}-\Sigma_{\exp }^{(2)}+\frac{1}{2}\left\{\left(\Sigma_{\exp }^{(1)}\right)^{2}-\left(\Sigma_{\mathrm{FO}}^{(1)}\right)^{2}\right\}\right)\right] } \\
& +\log \Sigma_{\mathrm{res}},
\end{aligned}
$$

resulting in

$$
\Sigma\left(\tau_{\mathrm{SD}}\right)=\Sigma_{\mathrm{res}} \exp \left[\frac{\alpha_{s}}{\pi}\left(\Sigma_{\mathrm{FO}}^{(1)}-\Sigma_{\exp }^{(1)}\right)+\frac{\alpha_{s}^{2}}{\pi^{2}}\left(\Sigma_{\mathrm{FO}}^{(2)}-\Sigma_{\exp }^{(2)}+\frac{1}{2}\left\{\left(\Sigma_{\exp }^{(1)}\right)^{2}-\left(\Sigma_{\mathrm{FO}}^{(1)}\right)^{2}\right\}\right)\right] .
$$

Multiplicative matching will be the default choice and the variation between the two will be included in the theoretical uncertainty, cf. section 5. In order to ensure that the differential cross section for the resummation and expansion vanishes for the fixed-order kinematical end-point, the resummation is modified in accordance with [40, 41], cf. appendix B.3 for details.

\footnotetext{
${ }^{1}$ Although the study was limited to $z_{\text {cut }}=0.1$, ref. [34] showed that after matching to NLO the residual finite $z_{\text {cut }}$ corrections were very small.
} 
Transition-point treatment. We briefly want to discuss the treatment of the transition point that marks the boundary between the soft-drop regime of the thrust distribution and the ungroomed one. Let us consider a LO configuration, where a gluon is emitted off the quark-antiquark pair. For sufficiently large values of thrust, i.e. above the transition point $\tau_{\mathrm{SD}}=z_{\text {cut }} / 2+\mathcal{O}\left(z_{\text {cut }}^{2}\right)$, the emission is always hard enough not to be impacted by grooming. Above this transition point the LO distribution for soft-drop thrust coincides with plain thrust. We note that for $z_{\text {cut }}=1 / 3$ and $\beta=2$, at leading order, the transition point coincides with the kinematic end-point, meaning the soft-drop thrust calculation stretches over the full LO distribution. The all-order calculation also features a transition point at $z_{\text {cut }} / 2+\mathcal{O}\left(z_{\text {cut }}^{2}\right)$, while if we consider higher orders in the fixed-order expansion, this transition point smears out due to the multiple emissions.

In the study of ref. [19], the transition point for the all-order calculation was taken as described above. However, this had the undesirable consequence of introducing transitionpoint corrections that break factorisation between the global soft and soft-collinear functions. In this current analysis we exploit the fact that at NLL accuracy we can treat the transition point between soft-drop and plain thrust as being located at $z_{\text {cut }} 2^{\beta / 2}$, which corresponds to the transition point for soft collinear emissions. The transition point is only located at $z_{\text {cut }} / 2$ for wide-angle emissions. Since the cross term between logarithms of 2 and logarithms of soft wide-angle origin starts contributing at NNLL accuracy, we can make use of this treatment at NLL accuracy, thus avoiding the aforementioned complications. In addition to this change in transition point, we can introduce a transition-point uncertainty. If the resummation uncertainty $\left(x_{L}\right)$ is only included in logarithms of thrust and not in the logarithms of $z_{\text {cut }}$, the transition point gains a resummation uncertainty dependence. The details on this approach are presented in appendix E.

We also consider a further effect which was not taken into account in the analysis of ref. [19], namely the non-trivial interplay between multiple-emission corrections and the transition point. Let us consider the emission of two gluons in the transition region (kept by the grooming procedure) such that the resulting $\tau$ is above the transition point. Corrections can appear when (one of) the individual contributions of these two emissions to the total $\tau$ value is below the transition point. This formally NNLL correction becomes parametrically relevant close to the transition point and is calculated in detail in appendix B. ${ }^{2}$

In figure 1 the results of these analytical computations are presented. From left to right the value of $\beta$ is varied between $\{0,1,2\}$, whereas top to bottom shows different values of $z_{\text {cut }}=\{0.05,0.1,0.2,0.33\}$. The results are presented at LO, NLO and NLO+NLL using both matching schemes. First a good agreement between the two different matching schemes can be seen. Both the contributions from the fixed-order transition point near $z_{\text {cut }} / 2$ and the resummation transition point can be seen. For lower values of $z_{\text {cut }}$ there is still a significant contribution from the second transition point, which is significantly reduced for larger values of $z_{\text {cut }}$.

It is interesting to point out that soft-drop also affects the thrust distribution above the transition point. We have already talked about transition point corrections in the context

\footnotetext{
${ }^{2}$ This correction cures the discontinuity in NLL distributions at the transition point noted out e.g. in $[19,24]$.
} 
of our resummed calculation (see also appendix B). For a fixed-order calculation the softdrop condition impacts the thrust distribution at all values of thrust. Finally, additional soft emissions, such as the ones related to the non-perturbative model, are also affected by grooming even above the transition point.

\section{Monte-Carlo based pseudo data}

Given that we attempt to extract the strong coupling from an observable where there exists no actual measurement yet, we have to resort to simulated data. Furthermore, we use Monte Carlo simulations to assess hadronisation corrections and the associated uncertainties.

We employ the Sherpa event generator $[25,26]$ version 2.2 .5 to simulate $e^{+} e^{-} \rightarrow$ hadrons events at LEP1 energy of $\sqrt{s}=m_{Z}$. We generate the hard-scattering configurations for varying parton-multiplicity final states at next-to-leading order in QCD. The matrix elements get matched to the Sherpa dipole parton shower [42] based on the Sherpa implementation of the MC@NLO method $[43,44]$ and merged into inclusive samples according to the MEPS@NLO formalism [45]. The required one-loop virtual amplitudes are obtained from the OpenLoops-1.3.1 [46] package. The default hadronisation model in Sherpa is the cluster fragmentation described in [47]. However, Sherpa also provides the option to invoke the Lund string fragmentation [48] as implemented in Pythia 6.4 [49]. Using the identical perturbative inputs, i.e. shower-evolved events, this provides us with a consistent estimate for the hadronisation related uncertainties. To analyse events we employ the Rivet-2.7.2 package [50]. To this end we have implemented the soft-drop thrust observable using FastJet-3.3.2 [31] for particle clustering.

For the generation of our pseudo data, used in the extractions of $\alpha_{s}$ later on, we consider the highest accuracy Monte Carlo sample which is generated using NLO QCD matrix elements for $e^{+} e^{-} \rightarrow 2,3,4,5$ partons. The MEPS@NLO merging parameter is set to $y_{\text {cut }}=\left(Q_{\text {cut }} / E_{\mathrm{CMS}}\right)^{2}=10^{-2}$. We evolve the strong coupling at the two-loop order, assuming $\alpha_{s}\left(m_{Z}\right)=0.117 .{ }^{3}$ While for the cluster fragmentation model all parameters are kept at their default values, we have set the main parameters of the Lund model to

$$
a=0.3(\operatorname{PARJ}(41)), \quad b=0.6 \mathrm{GeV}^{-2}(\operatorname{PARJ}(42)), \quad \sigma=0.36 \mathrm{GeV}(\operatorname{PARJ}(21)) .
$$

Comparisons of Sherpa MEPS@NLO hadron-level predictions with LEP1 event-shape data have for example been presented in [51]. To validate our event simulations, we present in figure 2 the plain thrust distribution as measured by ALEPH [52]. Shown there are the MEPS@NLO parton-level prediction, i.e. after parton showering, and hadron-level results for the cluster and Lund fragmentation model. Furthermore, the purely perturbative $\mathrm{NLO}+\mathrm{NLL}$ resummed prediction with an estimate of the perturbative uncertainty, indicated by the red band, cf. section 5, is given. The upper ratio plot compares theoretical predictions with the experimental measurement. Apart from the first bin both hadron-level

\footnotetext{
${ }^{3}$ The Sherpa default value is $\alpha_{s}\left(m_{Z}\right)=0.118$. However, we observed a marginally better description of LEP1 observables, and in particular thrust, both for the cluster and the Lund string fragmentation using $\alpha_{s}\left(m_{Z}\right)=0.117$.
} 
Soft-drop thrust distribution $\left(\frac{\tau}{\sigma_{0}} \frac{d \sigma}{d \tau}\right)$ for different parameters

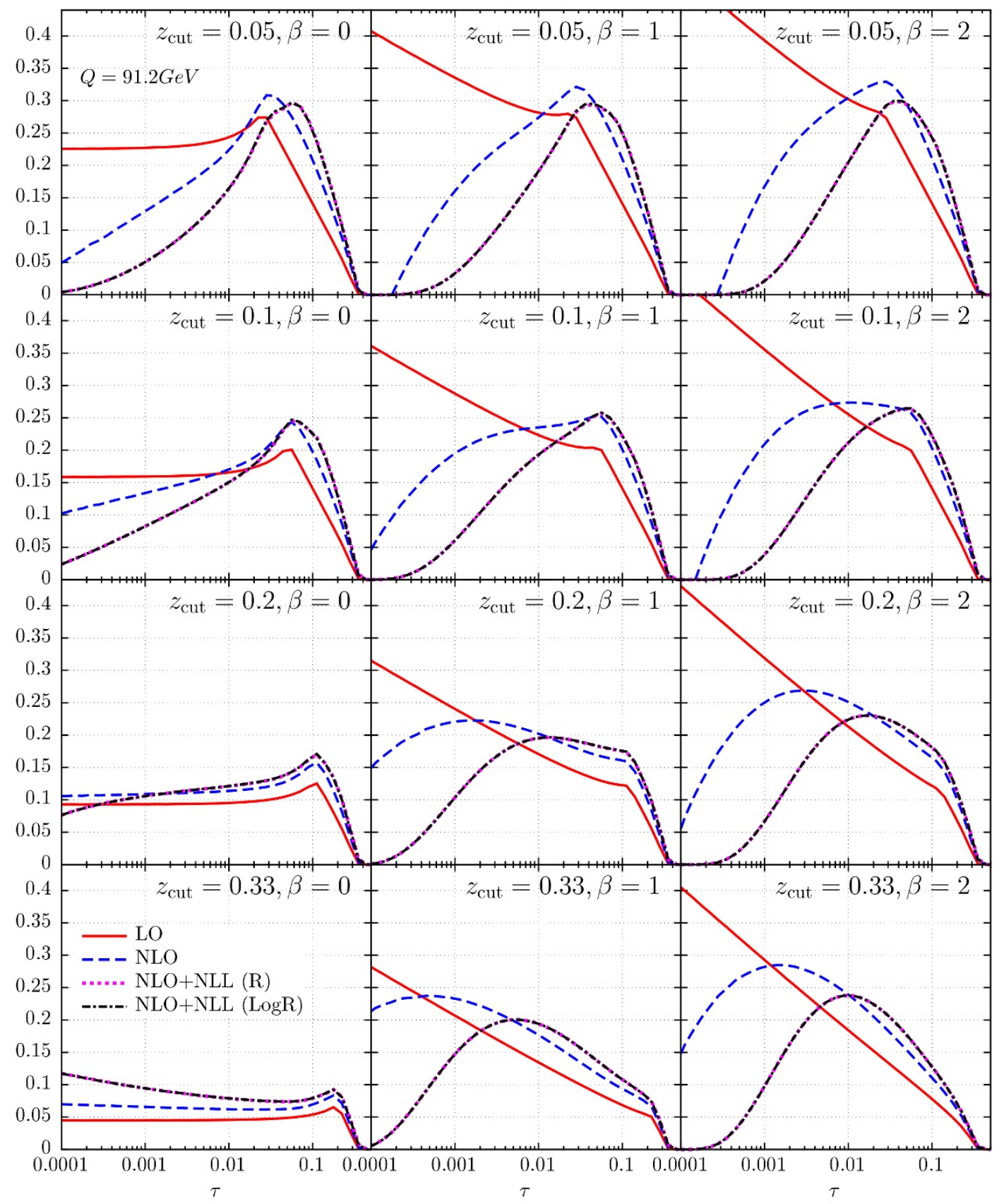

Figure 1. The differential cross section for the analytical soft-drop thrust distribution at LO (solid red), NLO (dashed blue) and NLO+NLL with both multiplicative (dotted magenta) and LogR (black dashed-dotted) matching. 


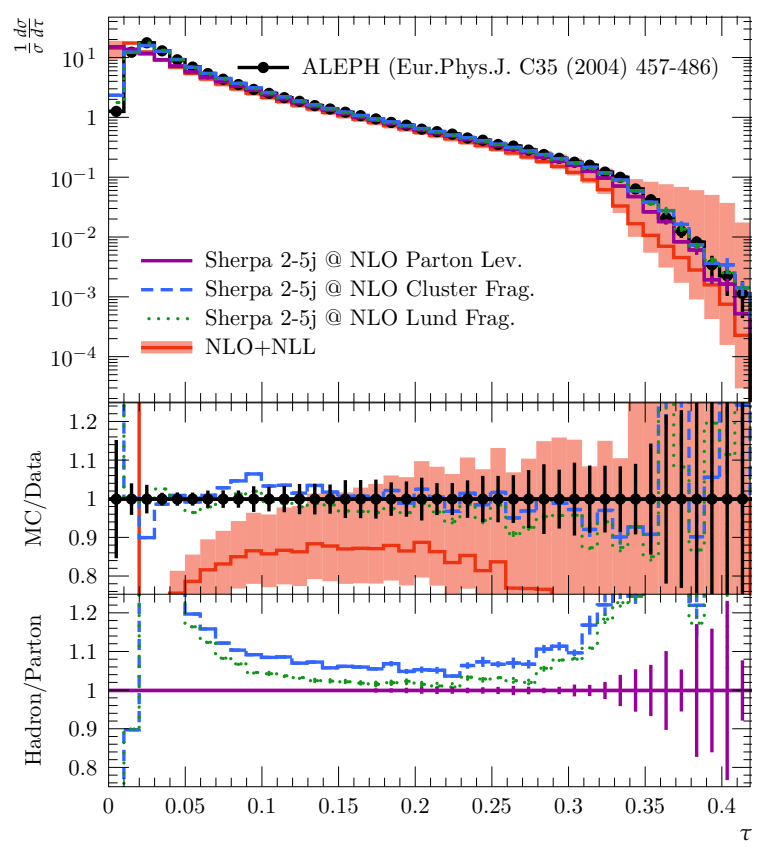

Figure 2. Plain thrust distribution as measured by ALEPH compared to Sherpa at parton level and with cluster and Lund fragmentation, and compared to the nominal resummed distribution matched to NLO. The lower panel shows the hadronisation corrections as obtained from Sherpa with the two fragmentation models considering MEPS@NLO with up to 5 jets at NLO.

results are in good agreement with data. However, the resummed calculation, without the inclusion of non-perturbative corrections, significantly undershoots the data, in particular for $\tau<0.1$.

The majority of this deviation originates from neglecting hadronisation effects in the analytic calculation. This is apparent from the lower ratio plot. Here the two hadronlevel results are compared with the parton-shower-level prediction. For $\tau \approx 0.05$ the hadronisation corrections are of order $20 \%$. In order to justify the direct extraction of hadronisation corrections for our analytic predictions of the soft-drop thrust observable we compiled Monte-Carlo simulations that better fit the fixed-order accuracy of the matched resummed calculations. For this purpose we only consider NLO QCD matrix elements for $e^{+} e^{-} \rightarrow 2,3$ partons in our Sherpa MEPS@NLO simulations, with the merging parameter still set to $y_{\text {cut }}=10^{-2}$. All other parameters are also kept unchanged. A dedicated comparison of Sherpa parton-shower simulations and NLL accurate predictions of some event-shape variables can be found in [53].

In figure 3 we present results for soft-drop thrust, considering $z_{\text {cut }}=$ $\{0.05,0.1,0.2,0.33\}$ (increasing from top to bottom) and $\beta=\{0,1,2\}$ (increasing from left to right). Besides the Sherpa parton-level predictions the respective hadron-level results for cluster and Lund fragmentation are given. Further, we show the NLO+NLL predictions. The lower panels contain the respective ratios with the corresponding parton-level MC predictions.

We begin with noting that the hadronisation corrections for soft-drop thrust are indeed reduced in comparison to plain thrust. As already seen in [19], the region where the 


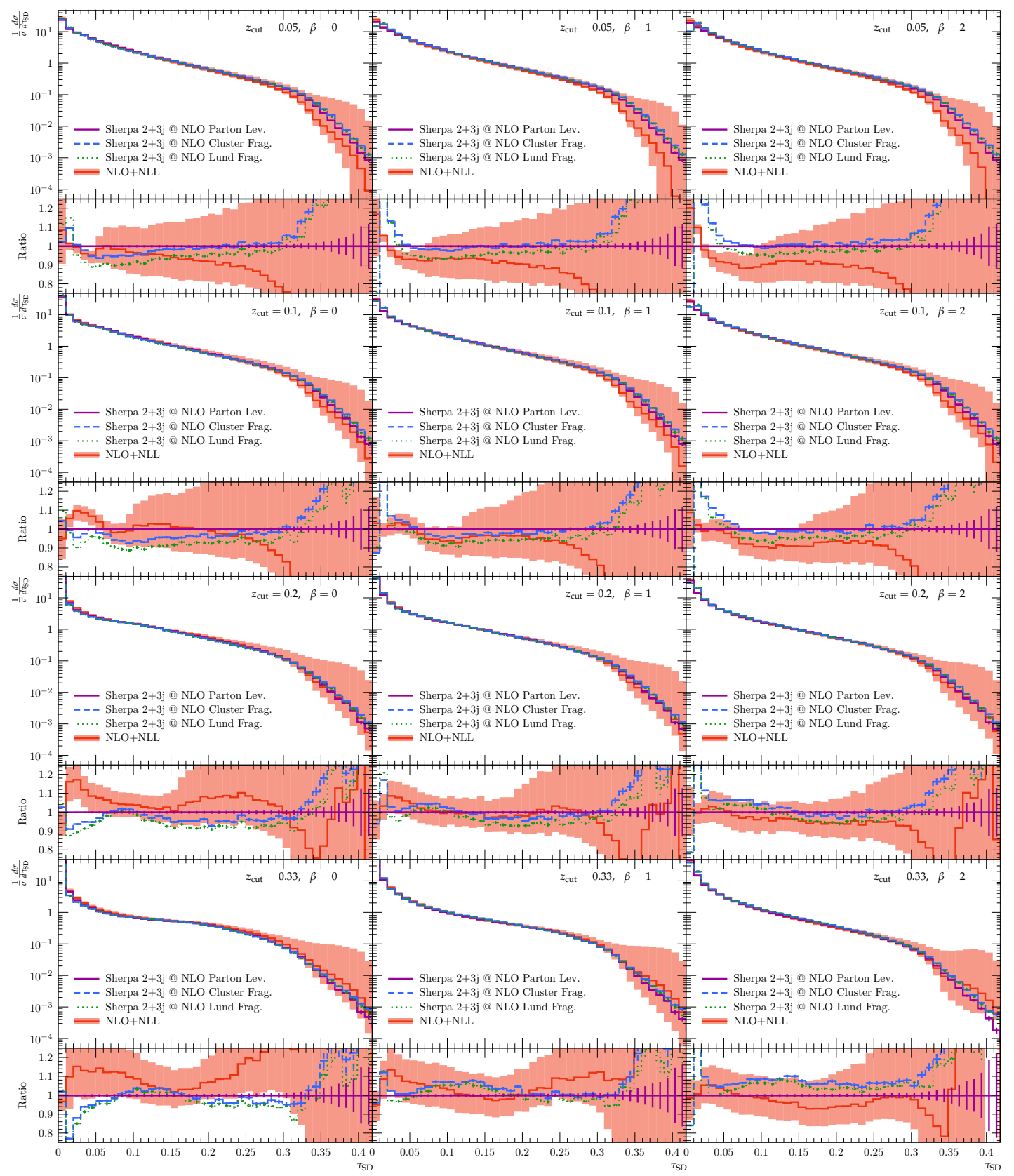

Figure 3. Comparison of NLO+NLL matched predictions for soft-drop thrust to Sherpa MEPS@NLO simulations with up to 3 jets at NLO at parton level and with cluster and string fragmentation applied.

hadronisation corrections are rather flat is extended towards smaller values of $\tau_{\mathrm{SD}}$. For all values of $z_{\text {cut }}$ and $\beta$ the shape of the corrections from the cluster and Lund string model are very similar. In particular for $z_{\text {cut }}=0.33$ both hadron-level predictions agree very well also in their nominal size. However, for the other $z_{\text {cut }}$ values the differences remain in the few percent range. 
Besides the size of the MC hadronisation corrections to be used later in the fits, figure 3 contains the direct comparison of the NLO+NLL calculations to the Sherpa parton-level prediction. For a wide range of the observables the agreement is well within $\pm 10 \%$, the ratio between both calculations being rather flat. Both results are certainly consistent within the inherent uncertainties. Note that, in contrast to the analytic NLO+NLL prediction, the shower result accounts for additional effects such as momentum conservation and finite recoil [53]. In appendix D further validation results are provided. We compare Sherpa results against hadron-level predictions from Pythia [54] and Herwig [55]. Furthermore, we also compare to the Dire [56] algorithm for parton showering within Sherpa in conjunction with the default cluster model for hadronisation.

\section{Hadronisation corrections}

Prior to describing our actual fits, we still need to address the issue of non-perturbative corrections from the parton-to-hadron transition. As mentioned before, this is crucial for fits performed with the plain thrust distribution, as perturbation theory alone is not able to reproduce the experimental data in the fitting range. We have seen that the situation is greatly ameliorated if we employ the soft-drop version of thrust. However, although reduced in size, hadronisation corrections play a non-negligible role also for soft-drop thrust.

To supplement resummed and matched calculations with non-perturbative hadronisation corrections, two main approaches can be found in the literature. On the one hand, analytical models of hadronisation can be constructed, based on fairly general physical assumptions and depending on one or few input parameters only. On the other hand, one can exploit the hadronisation models used in Monte Carlo events generators to extract a numerical estimate of hadronisation corrections by considering bin-by-bin ratios of the hadron-level and parton-level distribution. In the case of plain thrust, analytic models were used, for instance, in refs. [13-16, 18], ${ }^{4}$ while examples of fits of $\alpha_{s}$ exploiting Monte Carlo based hadronisation models can be found in refs. [8-12, 57].

\subsection{Monte-Carlo hadronisation model}

Analytical estimates of non-perturbative corrections in the presence of grooming are characterised by additional complexities, which require dedicated studies (see e.g. ref. [58] for a recent study). Therefore, in the current analysis, we have decided to resort to a MonteCarlo based approach as our default model for hadronisation corrections. To this end the ratios of hadron- to parton-level distributions are considered. The hadronisation models implemented in general-purpose Monte Carlo event generators depend on various parameters, whose values get tuned to data [59]. Hence, hadronisation corrections obtained from Monte Carlo simulations inevitably include some perturbative contribution from missing higher-order corrections that are specific to the underlying parton-level. Therefore, if we are to supplement our analytic calculations with hadronisation corrections extracted from Monte Carlo, a decent agreement with the parton-level Monte Carlo prediction should be in

\footnotetext{
${ }^{4}$ Note that $[14,16,18]$ make use of a shape function approach including renormalon subtraction, which is more advanced than the approach presented in this work.
} 
place. These comparisons were presented in section 3 for the Sherpa results at MEPS@NLO $2+3 \mathrm{j}$ level. There it is evident that the agreement is significantly improved for soft-drop thrust in contrast to plain thrust. This makes the use of Monte-Carlo based hadronisation corrections more viable for soft-drop thrust at the current accuracy.

As the actual hadronisation corrections we take the hadron-to-parton ratios extracted from the Sherpa MEPS@NLO 2+3j predictions, shown in figure 3. We consider the difference between the results for the cluster model and the Lund string fragmentation as an estimate of the hadronisation related uncertainty. As illustrated in appendix D these two ratios provide a good coverage of the complete span found for different Monte Carlo event generators. In addition, we include a comparison of our default method to a bin-by-bin migration-matrix approach and find only small differences between the two at our accuracy.

\subsection{Analytical hadronisation model}

As an alternative, we present the general concepts behind the analytical hadronisation model that was applied to ungroomed event (and jet) shapes [60] and has been extended, more recently, to the case of mMDT [30] and soft drop [61]. Details on the specific calculations underlying the results presented here are given in appendix $\mathrm{C}$.

In general we consider an additional (single) non-perturbative emission, that is supposed to be soft. For emissions below an infrared factorisation scale $\mu_{I}$ the perturbative coupling is replaced by a universal, non-perturbatively defined, finite quantity. When properly subtracting all perturbative contributions, one can thus estimate a hadronisation contribution to the observable. For plain thrust this results in a shift of the observable value:

$$
\delta \tau(\Omega)=2 \frac{\Omega}{Q},
$$

with $Q$ the centre of mass energy. The shift thereby is parametrised by the non-perturbative parameter

$$
\Omega \equiv C_{F} \Lambda\left(\mu_{I}\right)=C_{F} \int_{0}^{\mu_{I}} \frac{d k_{t}}{\pi} \delta \alpha_{s}\left(k_{t}\right)
$$

that needs to be fitted simultaneously with $\alpha_{s}$.

For soft-drop thrust the situation is slightly more complicated. Typically, a nonperturbative emission will be soft enough to be groomed away and thus has no impact on the observable value. However, this does not hold for a sufficiently collinear emission, with a low enough transverse momentum to be called non-perturbative but energetic enough to survive grooming. These types of contributions are suppressed in $k_{t}$ and will depend on a separate non-perturbative parameter, that is a function of $\beta$, cf. appendix C.2. However, in the region we are interested in, the dominant hadronisation correction to soft-drop thrust originates from a different configuration, namely a non-perturbative emission that survives grooming as it is protected by hard emissions through the clustering history. Accordingly, the non-perturbative emission must lie within an angular cone determined by the thrust value. The corresponding shift of the thrust distribution is calculated in the appendix and reads

$$
\delta \tau(\Omega)=\tau^{1 / 2}\left\langle(z(1-z))^{-1 / 2}\right\rangle \frac{\Omega}{Q},
$$


where the average is calculated using as weight the appropriate QCD splitting function. Note that the integral involved in the above average is sensitive to the transition point, as is described in detail in appendix C.2.

In addition to shifts in the value of thrust, there is a second way non-perturbative corrections affect soft-drop observables. Specifically, after radiating a non-perturbative emission the energy of one of the two hard subjets found by the soft-drop procedure more precisely the softer one - can be reduced so that it fails the soft-drop condition and gets groomed away. This energy shift can be taken into account in the form of a shift in the grooming parameter $z_{\text {cut }}$ :

$$
\delta z_{\mathrm{cut}}(\Omega)=\frac{C_{A}}{C_{F}}\left(\frac{Z_{\mathrm{SD}}\left(1-Z_{\mathrm{SD}}+\tau\right)}{2 \tau}\right)^{\beta / 2} \frac{\left(Z_{\mathrm{SD}}-\tau\right)\left(1-Z_{\mathrm{SD}}\right)-\tau}{\sqrt{\tau\left(Z_{\mathrm{SD}}-\tau\right)\left(1-Z_{\mathrm{SD}}\right)}} \frac{\Omega}{Q},
$$

where $Z_{\mathrm{SD}}=z_{c}^{2 /(2+\beta)}(2 \tau)^{\beta /(2+\beta)}$. Because at LO it is not possible for an emission to be groomed away above transition point, we freeze this correction in that region.

The $\Omega$ parameter for both the groomed thrust shift and $z_{\text {cut }}$ shift have the same corresponding integral definitions and approximations in their derivations, and are therefore assumed equal. While we use the same formal definition of the parameter $\Omega$ in the case of plain and soft-drop thrust, different approximations were assumed in the derivation of the respective hadronisation corrections and hence their numerical (fit) values are not expected to be identical. The model builds in the assumption that the observable and energy shifts are not too large and therefore the value of $\Omega$ is also assumed rather small. Accordingly, we restrict our fits to $\Omega \in[0,2] \mathrm{GeV}$.

The variation of plain thrust and soft-drop thrust under different assumptions for the non-perturbative parameter $\Omega$ is illustrated in figures 4 and 5 , respectively. For plain thrust the analytical hadronisation model produces hadronisation corrections very similar in shape ${ }^{5}$ to those extracted from Monte Carlo, cf. figure 2, though differences between parton level and resummation still cause different, but compatible, fitted values of $\alpha_{s}$. For equal values of $\Omega$, the hadronisation contribution to soft-drop thrust is significantly reduced. However, the behaviour for the analytical model does not fully replicate the hadron-to-parton ratios from Monte Carlo, cf. figure 3. For $\beta=0$ below the transition point $\left(z_{\text {cut }} / 2\right)$ the decrease in cross section corresponds to the behaviour seen in the Monte Carlo results. However, above the transition the $z_{\text {cut }}$ shift has little effect and therefore the thrust shift dominates. This leads to an increase in the cross section, which does not correspond to what is seen in the Monte Carlo simulations.

Recently a more refined computation of non-perturbative corrections to the groomed jet mass for $e^{+} e^{-}$colliders was performed [58]. This calculation can potentially be applied to the soft-drop thrust observable. However, as stressed in ref. [58], the calculation is only applicable below the transition point and, in particular, it does not reduce to

\footnotetext{
${ }^{5}$ Note that, even if they are similar in shape, analytic and Monte-Carlo-based hadronisation corrections can differ significantly. For example, analytic hadronisation corrections can be made arbitrarily small by taking $\Omega \rightarrow 0$, while Monte-Carlo-based corrections are bounded around what is given by the different generators.
} 


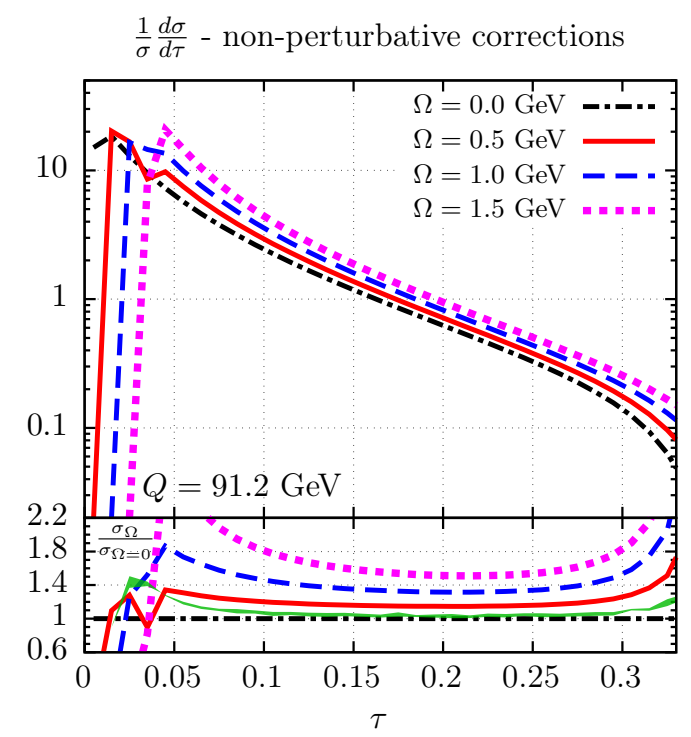

Figure 4. Comparison of the plain thrust NLO+NLL cross section under variation of the analytical hadronisation parameter $\Omega$. The lower panel displays the ratios with respect to the case of vanishing non-perturbative corrections, i.e. $\Omega=0 \mathrm{GeV}$ and the green band showing the hadron to parton level ratio for comparison.

the standard, i.e. ungroomed, non-perturbative correction. Since the region neighbouring the transition point is quite relevant to the fit, further developments are needed to employ a field-theoretical description of hadronisation corrections in precision fits of the strong coupling.

\section{Fitting procedure}

Having presented the theoretical calculations we are going to use for the determination of the strong coupling, as well as the Monte Carlo tools employed to generate pseudo data and our approaches to account for hadronisation corrections, we are now ready to discuss the actual fitting procedure. The fits are based on a prescription similar to what has been done for plain thrust, making use of a $\chi^{2}$ minimisation.

Fitting setup and uncertainty definition. The fit of $\alpha_{s}$ is performed by minimising the $\chi^{2}$ given by:

$$
\chi^{2}=\sum_{i, j}\left[\left(\frac{1}{\sigma} \frac{d \sigma}{d \tau}\left(\tau_{i}\right)\right)_{\exp }-\left(\frac{1}{\sigma} \frac{d \sigma}{d \tau}\left(\tau_{i}\right)\right)_{\mathrm{th}}\right] V_{i j}^{-1}\left[\left(\frac{1}{\sigma} \frac{d \sigma}{d \tau}\left(\tau_{j}\right)\right)_{\exp }-\left(\frac{1}{\sigma} \frac{d \sigma}{d \tau}\left(\tau_{j}\right)\right)_{\mathrm{th}}\right],
$$

where both the experimental and theoretical thrust distributions are normalised to the respective inclusive cross section and the thrust distribution in a bin of width $d \tau=0.01$ is computed using differences of the cumulative distribution $\Sigma(\tau)$ at the edges of the bin. The sums in the definition of $\chi^{2}$ extend over the considered observable bins. The correlation matrix $V_{i j}$ contains the uncertainties based on the experimental data

$$
V_{i j}=\delta_{i j} \sigma_{\mathrm{stat}}^{2}+\min \left(\sigma_{\mathrm{sys}, i}^{2}, \sigma_{\mathrm{sys}, j}^{2}\right)
$$




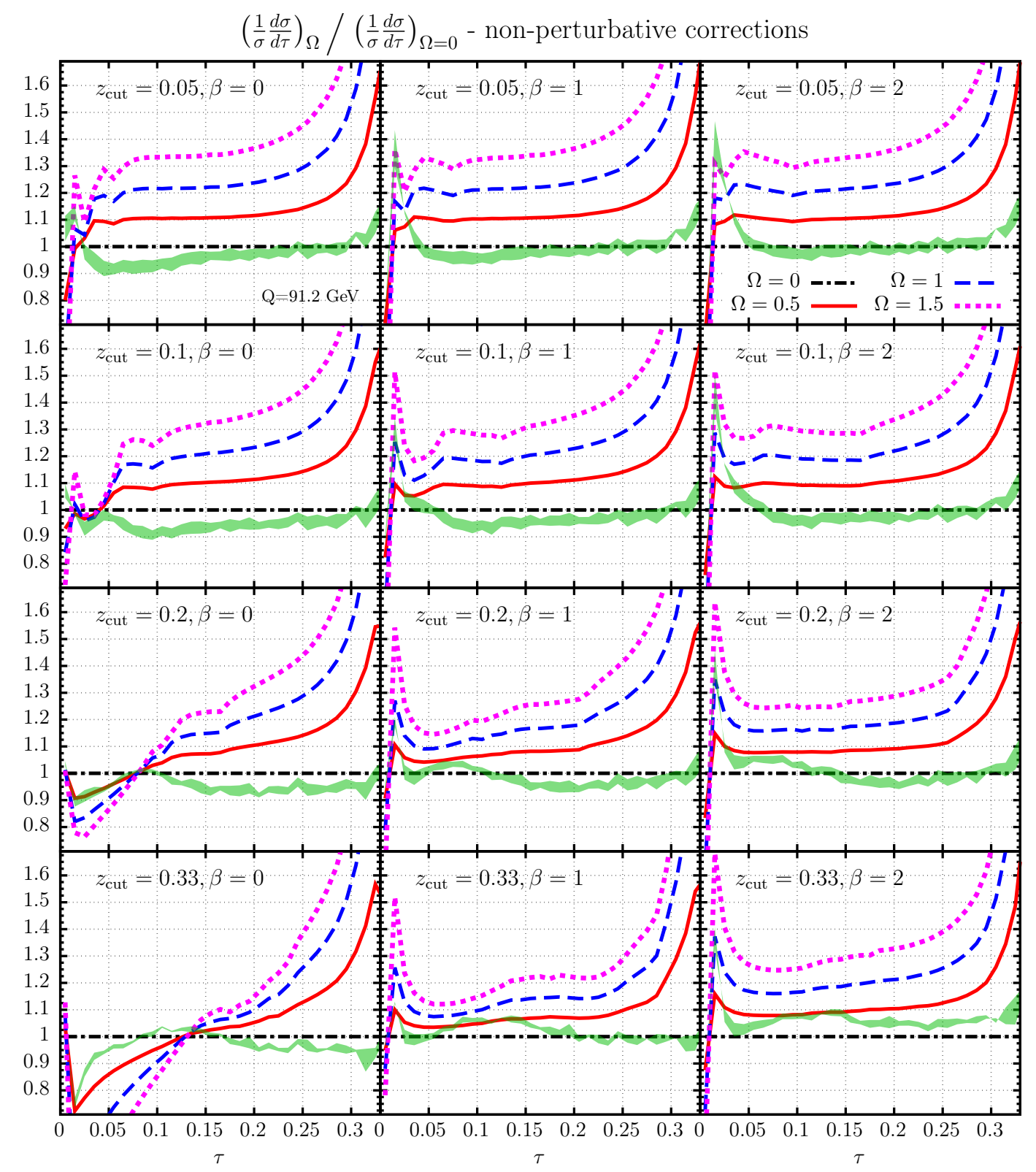

Figure 5. Ratios for the soft-drop thrust NLO+NLL cross section under variation of the analytical hadronisation parameter $\Omega$ with respect to no non-perturbative corrections, i.e. $\Omega=0 \mathrm{GeV}$. Where the green band shows the hadron to parton level ratio for comparison.

as assumed for previous fits $[14,15,18]$. The uncertainties for our Sherpa pseudo data are taken as directly proportional to the plain thrust ALEPH uncertainties:

$$
\begin{aligned}
\sigma_{\text {stat }}^{\mathrm{MC}} & =\sigma_{\text {stat }}^{\mathrm{ALEPH}} \sqrt{\frac{d \sigma^{\mathrm{MC}} / d \tau}{d \sigma^{\mathrm{ALEPH}} / d \tau}}, \\
\sigma_{\mathrm{sys}}^{\mathrm{MC}} & =\sigma_{\mathrm{sys}}^{\mathrm{ALEPH}} .
\end{aligned}
$$

The strong coupling constant is fitted for the central renormalisation scale choice $\mu_{R} / Q=1$. The resummation scale, which appears in the logarithms of $\tau$ and $z_{\text {cut }}$ (cf. 
appendix B.1), is also used at its central value $\mu_{Q} / Q \equiv x_{L}=1$. Finally multiplicative matching is used and the power for the end-point correction is $p=1$ [41] (cf. appendix B.3). When the Monte Carlo based hadronisation model is applied, the central fit is made using the average of the ratios of cluster and Lund string fragmentation to the parton-level prediction.

The experimental uncertainty on the central results is determined by the range of $\alpha_{s}$ values with $\Delta \chi^{2}=1$ around the central value while keeping the hadronisation parameter $\Omega$ fixed. The theory uncertainty is determined by varying simultaneously the theory inputs, i.e. the parameter $p$ assuming $p=1$ and 2, the matching scheme by switching between the multiplicative and $\log \mathrm{R}$ prescription and by 7 -point variations of the perturbative scales (excluding $\mu_{R} / Q=x_{L}=2$ and $\mu_{R} / Q=x_{L}=1 / 2$ ) using the best fit for each of these variations. The corresponding uncertainty is then defined by the difference between the central value fit and the found minimum and maximum variations in the fitted value of $\alpha_{s}$ (and $\Omega$ ).

The hadronisation uncertainty is model dependent. For the analytical model the uncertainty is determined by $\Delta \chi^{2}=1$ as above while allowing for a variation of $\Omega$. The previously mentioned experimental uncertainty needs to be subtracted from this quadratically to prevent double counting. For the Monte-Carlo based model the hadronisation uncertainty is determined by the range between the best fit using either the cluster model or the Lund string fragmentation.

\section{Results}

In this section we present our results for the extraction of $\alpha_{s}$ from fitting NLO+NLL predictions for the soft-drop thrust observable to Monte-Carlo pseudo data. To account for the parton-to-hadron transition we employ both the analytical approach and Monte-Carlo simulations. We are particularly interested in assessing the impact of soft-drop grooming on the stability of the fits and their quality. To this end we compare results obtained for soft-drop thrust to those for plain thrust.

The default observable range used in the fits is $0.06 \leq \tau \leq 0.25$. The lower boundary equals the one used in the previous thrust fits, cf. [14, 18]. However, the upper boundary is somewhat reduced, as in our study we work at a lower fixed-order precision, i.e. NLO instead of NNLO QCD, resulting in a reduced accuracy for the distributions large- $\tau$ tail.

We begin by considering the stability of the extracted strong coupling under variations of the type of theoretical prediction entering the fit. In figure 6 we present results for the best-fit $\alpha_{s}\left(m_{Z}\right)$ value and the associated total and hadronisation related (shaded band) theoretical uncertainties. Besides the pure fixed-order result from EVENT2 [35, 36] (FO), we use the NLO+NLL resummed prediction matched to the NLO matrix element (Res), as well as this resummed result dressed with non-perturbative corrections from the MonteCarlo fragmentation approach (NP (MC)) and the analytic model (NP (ana)). We present results for three values of the soft-drop angular exponent $\beta=0,1,2$. For each value of $\beta$ we consider $z_{\text {cut }}=0.05,0.1,0.2,0.33$, indicated by different colours. Furthermore, for comparison, we present the results obtained for plain thrust in each plot. 

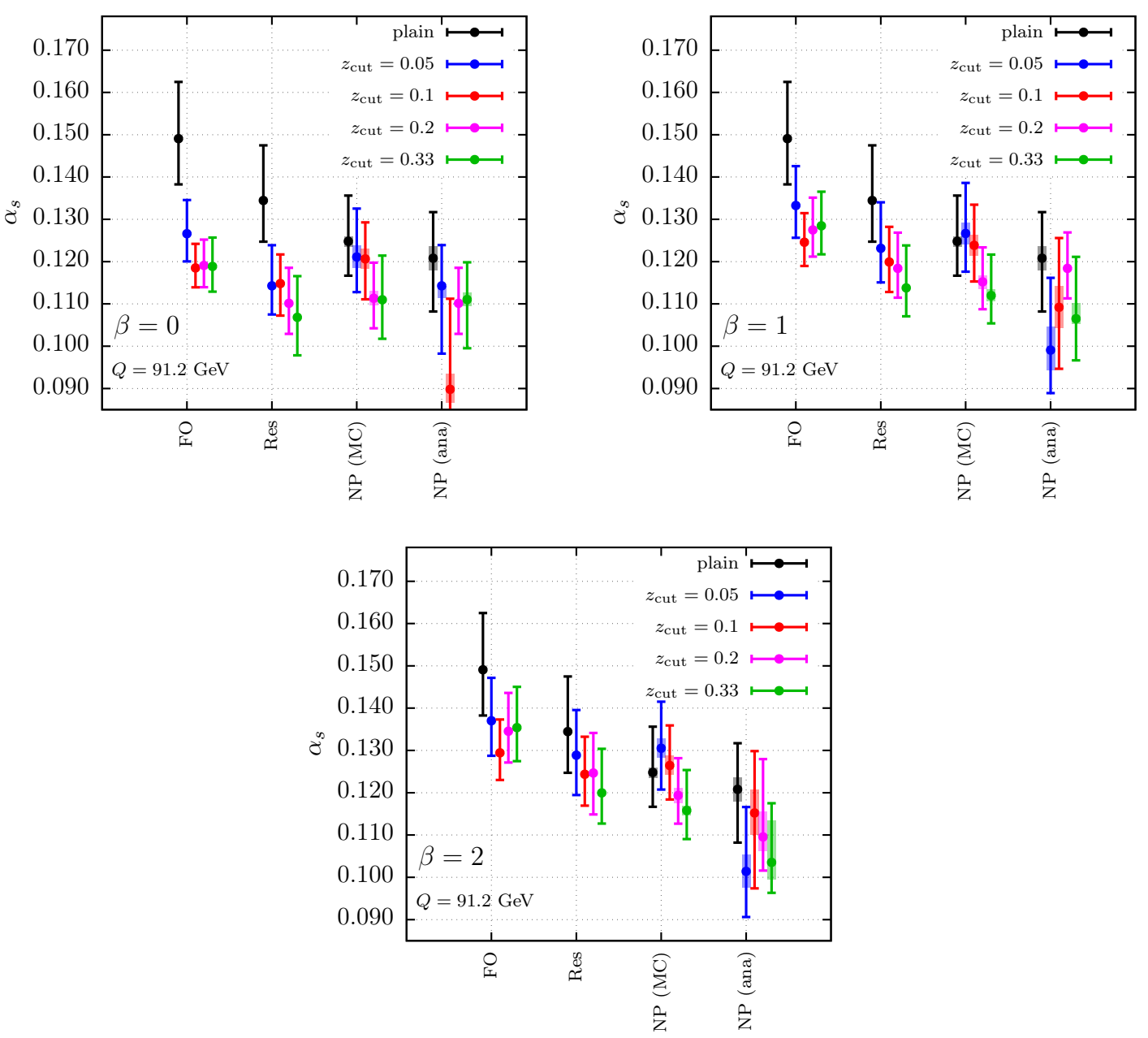

Figure 6. Results for fits of $\alpha_{s}\left(m_{Z}\right)$ to Monte-Carlo pseudo data using theoretical predictions at different levels: FO for NLO, Res for NLO+NLL and NP for the inclusion of non-perturbative effects. The non-perturbative effects are modelled based on either the Monte-Carlo based hadronto-parton level ratios (NP (MC)) or an analytical model with a single parameter $\Omega$ (NP (ana)). The bands indicate the total uncertainty, the shaded region displays the hadronisation-related uncertainty.

From the plots we can draw some first general conclusion. The impact of incorporating NLL resummation is sizeable both for the groomed and ungroomed observables. We observe a significant reduction of the best fit $\alpha_{s}\left(m_{Z}\right)$ in comparison to the fixed-order hypotheses. However, the role that non-perturbative corrections play is rather different. The shift induced by the inclusion of hadronisation corrections is significantly reduced when softdrop is employed. This is true for both the analytical and the Monte-Carlo based models for hadronisation. Unfortunately, this reduced impact of hadronisation corrections is not accompanied by a reduction of the associated uncertainties.

However, while these general patterns appear to be well-established, there are noticeable counterexamples in particular when using the analytical model for estimating hadro- 
nisation corrections. The first one standing out is $\beta=0$ and $z_{\text {cut }}=0.1$ (shown in red in the upper left plot of figure 6). For this particular set of soft-drop parameters the lower boundary of the fitting region is just above the LO transition point. However, in the analytic hadronisation model the transition point can, for large enough value of $\Omega$, be shifted into the fitting interval. This effect leads to an odd fitting behaviour. It is dominant for $\beta=0$ as in this case transition-point effects are more significant. Additionally, too little grooming such as $z_{\text {cut }}=0.05$ and $\beta>0$ (shown in blue in the upper right and lower plot of figure 6) shows a significant decrease in the fitted value of $\alpha_{s}$, when the analytic model of hadronisation is employed. An identical study for an independent resummation code with a different resummation uncertainty treatment, which allows for a shift in the transition point, is presented in appendix E.

All in all, our study suggests that a tighter grooming - i.e. larger values of $z_{\text {cut }}$, namely $z_{\text {cut }}=0.2$ and $z_{\text {cut }}=0.33$, or smaller values of $\beta$, namely $\beta=0$ or $\beta=1$ - shows a significant improvement compared to plain thrust with respect to both the shift in the fitted value due to the inclusion of hadronisation effects and the stability under variations of the hadronisation model. While the reduced shift from the analytical hadronisation corrections is clearly visible for the $\beta=0$ and $\beta=1$ cases, for $\beta=2$ this shift is qualitatively similar to the one observed for plain thrust. The results are further detailed in table 1. Besides the values obtained for $\alpha_{s}\left(m_{Z}\right)$ for the various scenarios considered, we provide the best-fit $\chi^{2}$-values, and, for the case of the analytic hadronisation model, the best-fit $\Omega$-parameter. For most of the groomed observables the $\chi^{2} /$ dof for the NLO+NLL matched calculation without any non-perturbative effects are already close to 1 . The only soft-drop parameter combinations that yield larger $\chi^{2}$ values are the two cases that were pointed out previously, $z_{\text {cut }}=0.1$ with $\beta=0$ and $z_{\text {cut }}=0.05$ with $\beta=2$. An important validation of our approach is the consistency between the value of $\alpha_{s}$ when fitting to ALEPH data instead of the pseudo-data for plain thrust. Additionally, it can be seen that despite the reduced data in the fitting region due to grooming the experimental uncertainty does not increase significantly, unless a significant amount of grooming is applied, for example $z_{\text {cut }}=0.33$ and $\beta=0$.

To better visualise the results of our study, we can directly compare our theoretical predictions for the thrust observable using the corresponding best-fit $\alpha_{s}$ with the (pseudo)data. In figure 7 we present this comparison for plain thrust. The left plot shows the result for the fit to the ALEPH data while the right plot shows those for the Monte-Carlo pseudo data instead. Both plots illustrate a very good agreement of analytic predictions and both the actual collider and the pseudo data over the whole fitting range. The red band represents the theoretical uncertainty on the fitted distribution for the analytical model. The differences to the distribution with hadronisation corrections based on the Monte Carlo model are small compared to the overall size of the uncertainty, so we choose not to include a separate error band for this to keep the plot clearer. It can also be seen that the behaviour in the small thrust region would be hard to reproduce using the analytic model. The corresponding results for soft-drop thrust, compared to our pseudo data, are presented in figure 8 , for all the $z_{\text {cut }}$ and $\beta$ values considered in this study. Again, a good agreement is found. For the considered fit range the biggest deviations are observed for 


\begin{tabular}{|c|c|c|c|c|c|c|c|c|c|c|c|c|c|c|c|c|}
\hline $\begin{array}{l}\stackrel{O}{\Sigma} \\
\frac{\partial}{z}\end{array}$ & 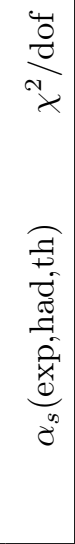 & 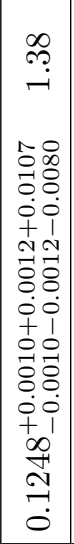 & 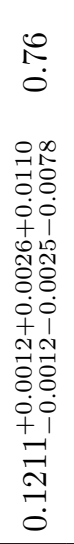 & 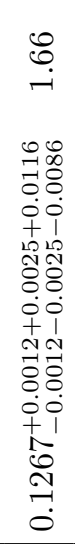 & 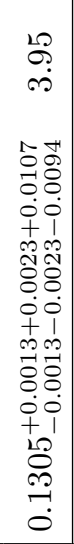 & 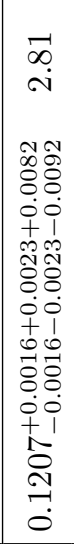 & 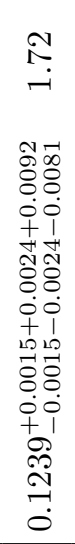 & 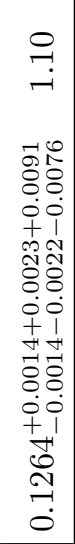 & 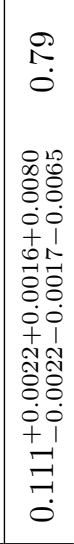 & 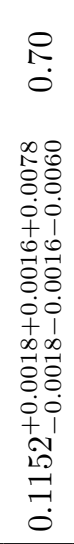 & 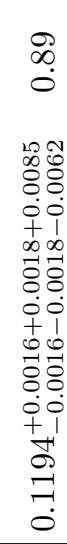 & 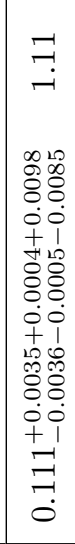 & 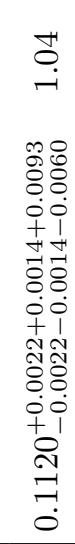 & 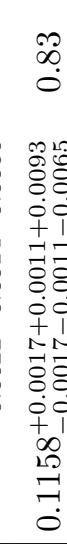 & 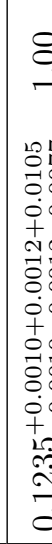 & \\
\hline 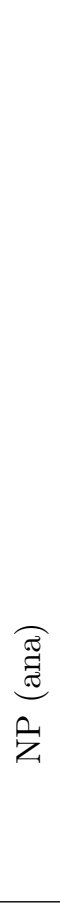 & 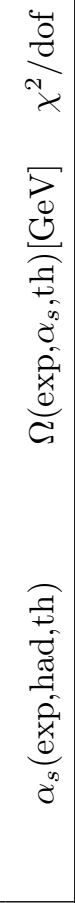 & 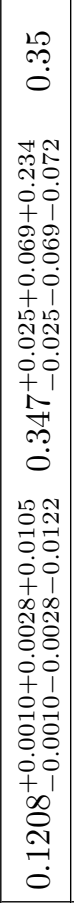 & 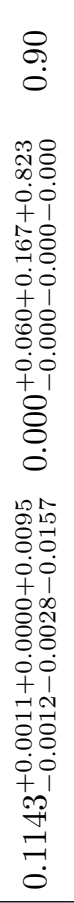 & 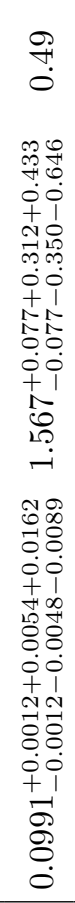 & 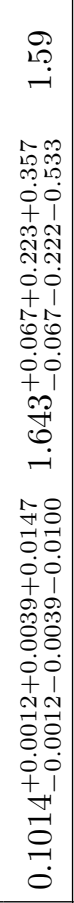 & 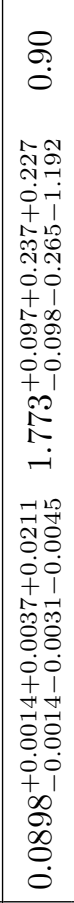 & 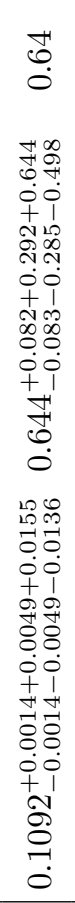 & 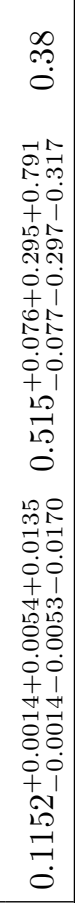 & 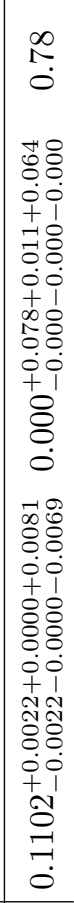 & 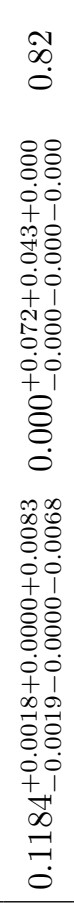 & 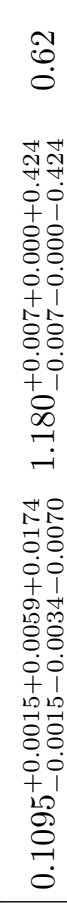 & 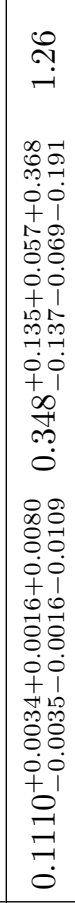 & 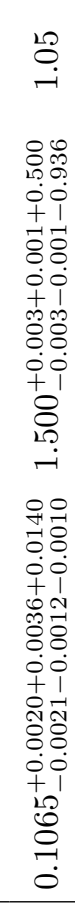 & 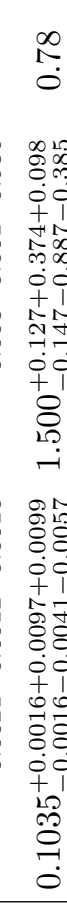 & 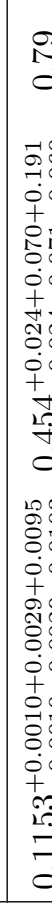 & \\
\hline$\underset{n}{n}$ & 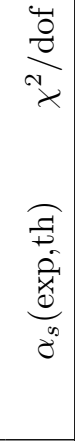 & 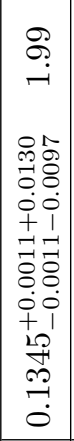 & 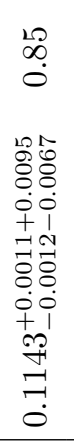 & 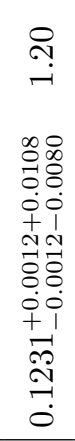 & 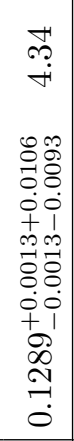 & 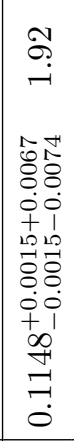 & 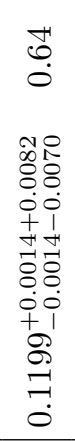 & 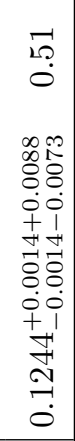 & 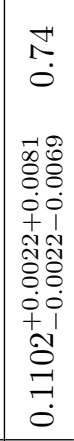 & 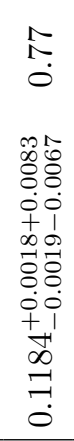 & 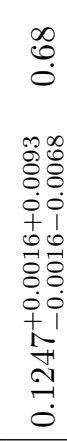 & 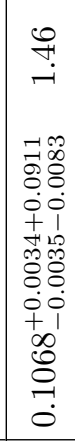 & 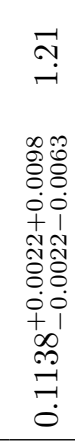 & $\begin{array}{l}0 \\
0 \\
0 \\
0 \\
0 \\
+1 \\
1 \\
01 \\
00 \\
0 \\
0 \\
+1 \\
0 \\
0 \\
0 \\
0\end{array}$ & 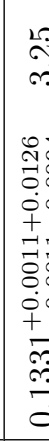 & \\
\hline & $\begin{array}{r}\overrightarrow{\vec{u}} \\
\text { v } \\
\end{array}$ & 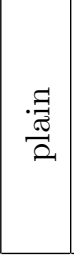 & $\begin{array}{l}0 \\
20 \\
0 \\
0\end{array}$ & $\neg$ & $N$ & $\overrightarrow{0}$ & - & $N$ & $\stackrel{\sim}{0}$ & $\neg$ & $\sim$ & $\ddot{0}$ & - & $\curvearrowright$ & & \\
\hline
\end{tabular}


large values of thrust when using the analytic model. The agreement in this region could potentially be improved by including NNLO fixed-order corrections, cf. [33]. When the Monte-Carlo based hadronisation model is used the most sizeable deviations are found in the centre of the fitting range, which may also be resolved by increasing the calculational accuracy. One also sees that the deviations are larger for the more problematic cases discussed above, $z_{\text {cut }}=0.1, \beta=0$ and $z_{\text {cut }}=0.05, \beta=2$, while they remain very small for $z_{\text {cut }}=0.2, \beta=0,1$.

All the results presented so far have been obtained performing the fits in the range that is typically employed in $\alpha_{s}$ extractions using plain thrust, i.e. $0.06 \leq \tau \leq 0.25$. In particular, the lower bound of this interval is a consequence of sizeable non-perturbative corrections for even smaller values of thrust, signalling the breakdown of the perturbative approach. However, a key observation of ref. [19] was that the impact of non-perturbative corrections on the soft-drop thrust distribution is consistently below $10 \%$ for a much wider observable range compared to plain thrust. Therefore, we can take advantage of this behaviour and push the lower bound of the fitting range to smaller values of $\tau$, while retaining perturbativity. This is a key property of soft-drop observables, which has been exploited also in the context of jet-mass measurements at the LHC [34, 61].

In order to quantitatively assess this observation, we perform several fits for the strong coupling reducing the lower bound of the fitting region. The results are reported in figure 9. As a measure of the fit quality, we present in the left column the resulting $\chi^{2} /$ dof values as a function of the lower bound of the fitting range $\tau_{\min }$, for plain thrust and the various soft-drop parameters. The fits are performed with the analytic model for the hadronisation corrections. It is apparent that the fit quality for plain thrust rapidly deteriorates below $\tau_{\min } \simeq 0.04$. Non-perturbative corrections become so large that they invalidate not only the perturbative approach but also the assumptions that go into the rather simple analytic model of hadronisation. Indeed the deterioration of the $\chi^{2} /$ dof is not as dramatic, when using the Monte-Carlo based model for hadronisation. However, the fit quality as measured by the $\chi^{2} /$ dof for soft-drop thrust depends very weakly on the value of $\tau_{\min }$ only. It is consistently better than what is obtained for plain thrust.

Similar conclusions can be drawn by looking at the right column of figure 9, where the best-fit $\alpha_{s}\left(m_{Z}\right)$ values are shown as a function of the lower bound of the fitting region $\tau_{\min }$. The best-fit $\alpha_{s}$ obtained with plain thrust exhibits a significant dependence on $\tau_{\min }$, while the values obtained with soft-drop thrust are rather stable under variation of $\tau_{\min }$. Furthermore, we remind the reader that in figure 6 we observed an issue for the fit performed with $z_{\text {cut }}=0.1$ with $\beta=0$, resulting in a rather small value of the strong coupling (with large uncertainties). We argued that this originates from the proximity of the transition point and the lower bound of the fitting range, causing an instability in the modelling. The top right plot in figure 9 fully supports this interpretation and indeed shows that the issue can be resolved by pushing the lower bound of the fitting range to smaller values. Choosing $\tau_{\text {min }} \lesssim 0.05$, we obtain fitted values of $\alpha_{s}$ that are compatible with all the other combinations of soft-drop parameters.

In addition to the fitted value of $\alpha_{s}\left(m_{Z}\right)$ and the value of $\chi^{2}$ one can investigate the dependence of the uncertainty on the increased fitting range. However, at the current theoretical precision the impact on the uncertainty is found to be not significant. 

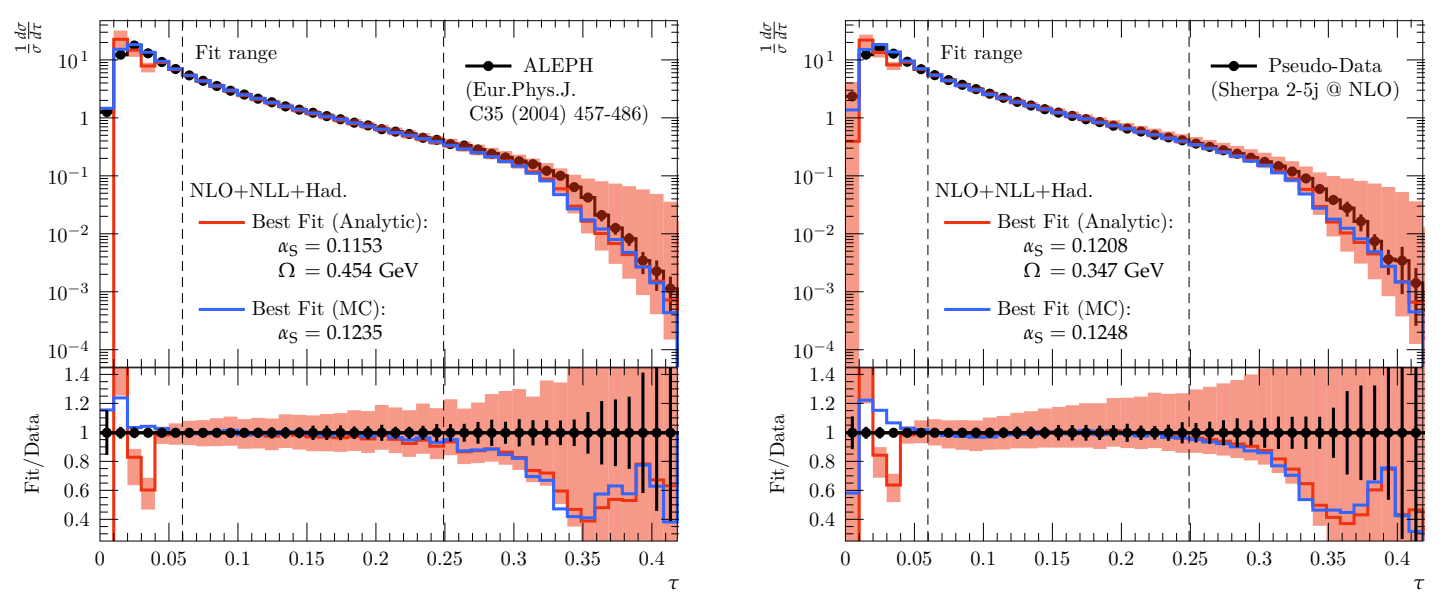

Figure 7. Plain thrust distribution as measured by ALEPH (left) and for Monte Carlo pseudo data (Sherpa MEPS@NLO 2-5j, right) compared to the NLO+NLL prediction with the best-fit values for $\alpha_{s}\left(m_{Z}\right)$ and the analytic (red) and Monte-Carlo (blue) hadronisation corrections. Uncertainties of the pseudo data are determined by rescaling of the uncertainties for the plain-thrust ALEPH data.

\section{Conclusion}

In previous work it has been shown that the soft-drop thrust observable features reduced sensitivity to non-perturbative effects. Motivated by this observation we have considered fits of the strong-coupling constant for this variable. We have analysed the impact of both resummation and hadronisation corrections and compared to plain thrust. Two different approaches to estimate hadronisation corrections have been used, one based on MonteCarlo results from Sherpa and another based on analytical computations.

The Monte-Carlo based model turns out more viable for soft-drop thrust than for plain thrust. This roots in an improved agreement of parton-level predictions based on NLO QCD matrix-element plus parton-shower simulations and the NLO+NLL analytical results. Through the use of this model we have shown a significant decrease in the shift of the extracted $\alpha_{s}\left(m_{Z}\right)$ due to hadronisation effects.

As an alternative an analytical hadronisation model for soft-drop thrust has been presented. Also here the general trend shows an improvement over plain thrust, however, some outliers have been noted. In particular lower values of $z_{\text {cut }}$ show issues in the fits related to the transition point. In addition, for the analytical model we have shown that it is viable to extend the fitting range to significantly lower values of the observable, which is not possible for plain thrust when using the analytical model. Despite these improvements for the analytic hadronisation model it still shows a relatively different behaviour compared to the Monte-Carlo based approach. This indicates the need for a more detailed analysis of the analytical model based on a more elaborate computation.

These conclusions were all made based on an analytical computation at NLO+NLL accuracy. However, for a future accurate measurement of the strong coupling this will need to be extended to at least NNLO+NNLL precision. The NNLO computation presented in [33] has been performed for the original definition of soft-drop thrust and could be 


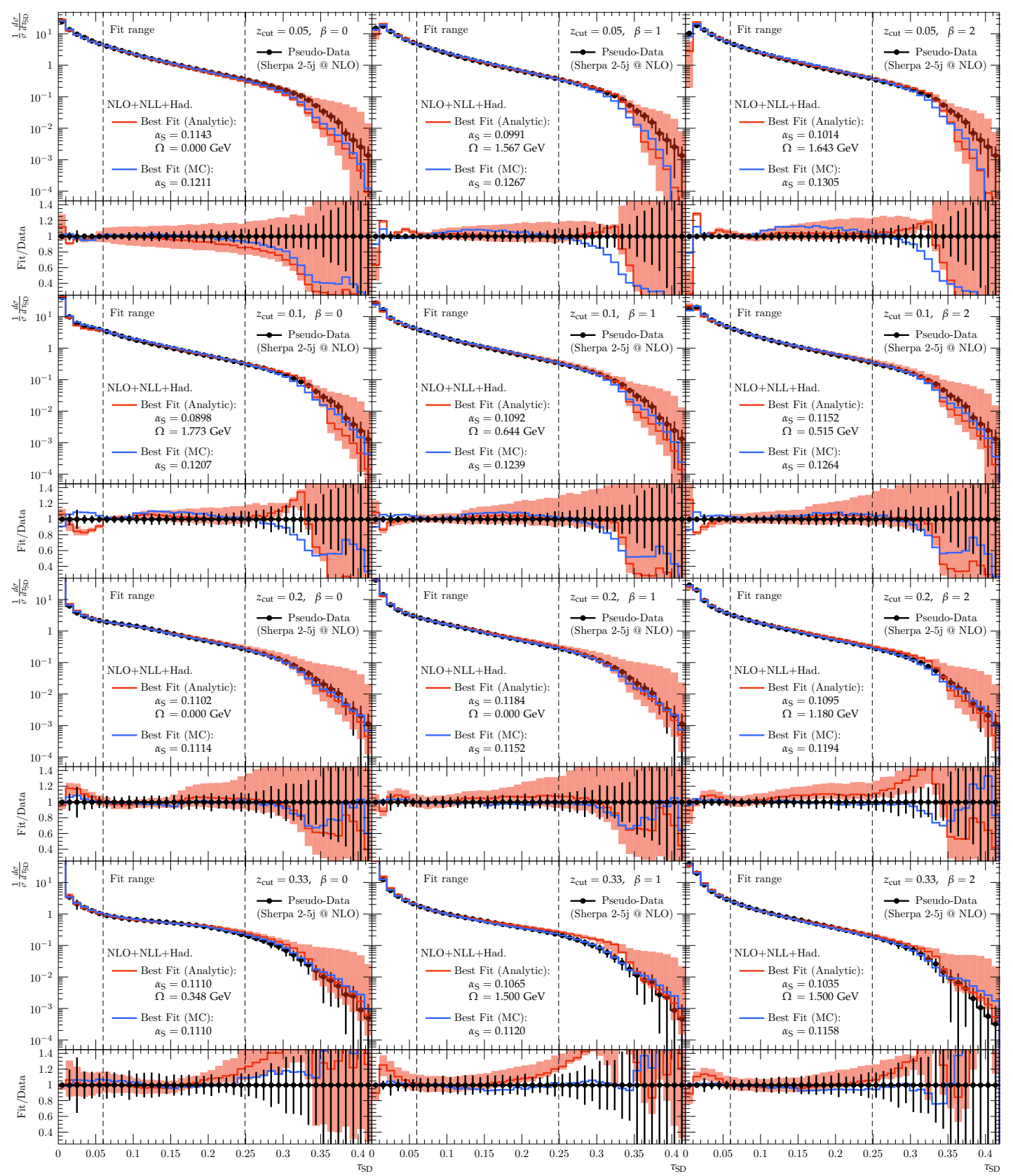

Figure 8. Pseudo-data (Sherpa MEPS@NLO 2-5j) and NLO+NLL result with best-fit values for $\alpha_{s}\left(m_{Z}\right)$ and the analytic (red) and Monte-Carlo (blue) hadronisation corrections. Uncertainties of the pseudo data are determined by rescaling of the uncertainties for the ALEPH ungroomed thrust data.

extended to the collinear-safe definition used in this work. The NNLL accuracy has been achieved in the limit $\tau \ll z_{\text {cut }} \ll 1$, however in the region relevant to this fit transitionpoint effects $\left(\tau \sim z_{\text {cut }}\right)$ will also need to be taken into account up to NNLL. Finally, due to the higher values of $z_{\text {cut }}$ used in this analysis the impact of finite $z_{\text {cut }}$ corrections (in particular for $\beta=0$ ) needs to be studied. 

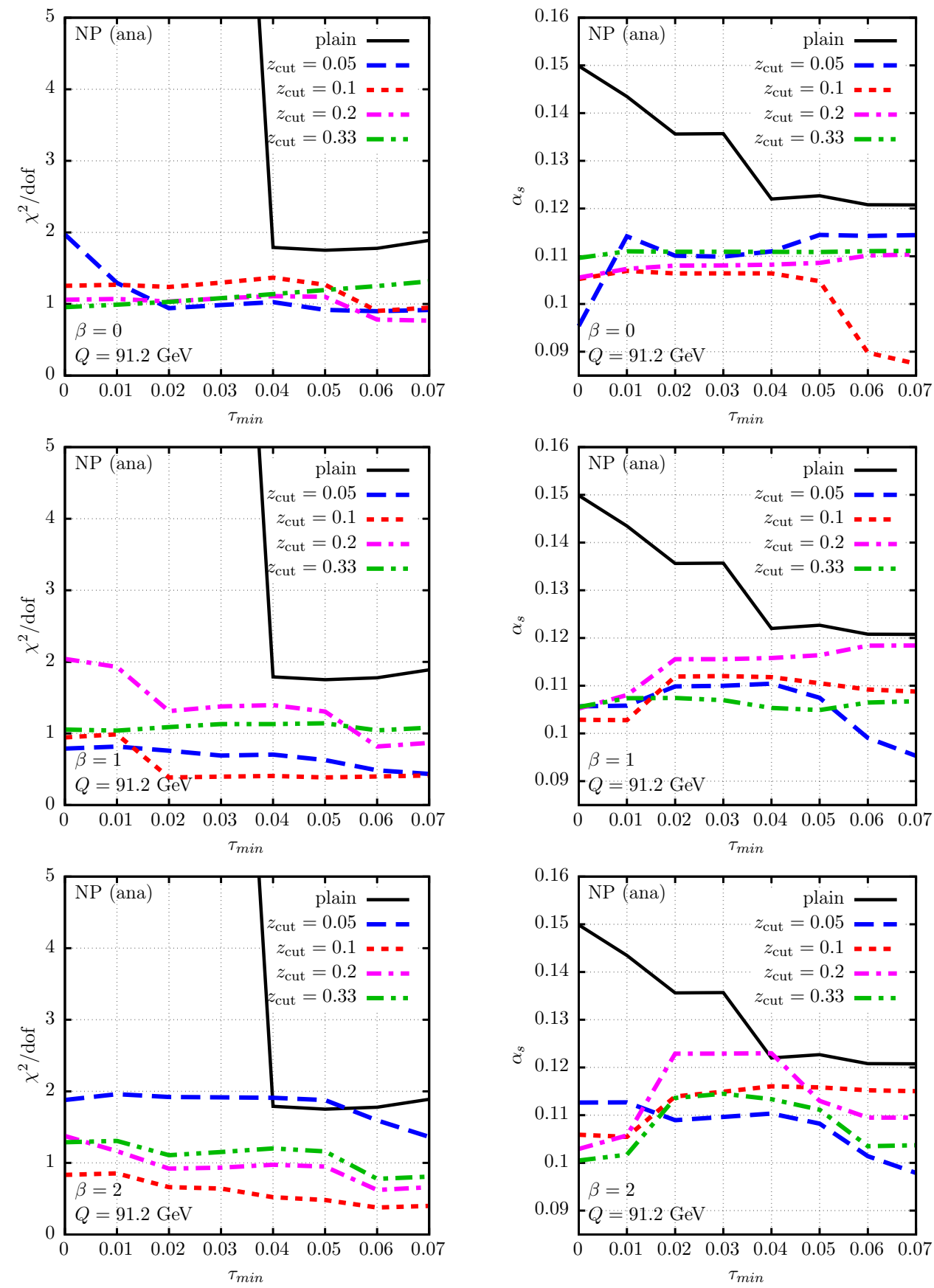

Figure 9. The best-fit $\chi^{2} /$ dof (left column) and $\alpha_{s}\left(m_{Z}\right)$ (right column) values as a function of the lower bound of the fitting range, $\tau_{\min }$. Shown are results for plain thrust and for soft-drop thrust, using different combinations of the soft-drop parameters $\beta$ and $z_{\text {cut }}$.

Finally, we note that, in order to reduce the impact of transition-point corrections and to simplify their computation, it would be worth considering other observables and modified definitions of the soft-drop grooming procedure itself. Similar studies can also be performed for event shapes at the LHC, where, besides hadronisation, in particular the underlying 
event obscures the comparison of perturbative predictions with actual collider data. In conclusion, the work presented here shows promising possibilities for the application of soft drop to the measurement of the strong-coupling constant. However, additional work will need to go into improving the theoretical accuracy to result in a precision extraction entering the world average.

\section{Acknowledgments}

We thank Pier Monni for the useful discussions. The work leading to this publication was supported by the German Academic Exchange Service (DAAD) with funds from the German Federal Ministry of Education and Research (BMBF) and the People Programme (Marie Curie Actions) of the European Union Seventh Framework Programme (FP7/20072013) under REA grant agreement n. 605728 (P.R.I.M.E. Postdoctoral Researchers International Mobility Experience). This work is also supported by the curiosity-driven grant "Using jets to challenge the Standard Model of particle physics" from Università di Genova. SS acknowledges funding from the European Union's Horizon 2020 research and innovation programme as part of the Marie Skłodowska-Curie Innovative Training Network MCnetITN3 (grant agreement no. 722104), the Fulbright-Cottrell Award and from BMBF (contract 05H18MGCA1). DR acknowledges support from the German-American Fulbright Commission allowing him to stay at Fermi National Accelerator Laboratory (Fermilab), a U.S. Department of Energy, Office of Science, HEP User Facility. Fermilab is managed by Fermi Research Alliance, LLC (FRA), acting under Contract No. DE-AC02-07CH11359. VT also thanks the CNRS and the IPhT for financial support and hospitality during the early stages of this work. GS is supported in part by the French Agence Nationale de la Recherche, under grant ANR-15-CE31-0016.

\section{A Collinear safety}

In this appendix we show that the rescaling factor in front of eq. (2.4) is crucial to make soft-drop thrust infrared-and-collinear safe for the modified Mass-Drop tagger (i.e. softdrop with $\beta=0)$.

Consider the situation where there is a single massless particle, of energy $E_{R}$ in the right hemisphere. The square bracket in eq. (2.4) is then

$$
\tau_{\mathrm{SD}}^{\text {(unscaled) }}=1-\frac{E_{R}+P_{L}}{E_{R}+E_{L}}=\frac{E_{L}-P_{L}}{E_{R}+E_{L}},
$$

with $P_{L}=\sum_{i \in \mathcal{H}_{\mathrm{SD}}^{L}}\left|\vec{n}_{L} \cdot \vec{p}_{i}\right|$ and $E_{L}=\sum_{i \in \mathcal{H}_{\mathrm{SD}}^{L}}\left|\vec{p}_{i}\right|$ the contributions of the left hemisphere to the numerator and denominator respectively (after soft-drop).

If the particle in the right hemisphere splits collinearly in two particles of energies $z E_{R}$ and $(1-z) E_{R}$ the softest of these two particles, say the one with energy $z E_{R}$, will be groomed away and the unscaled soft-drop thrust becomes

$$
\tau_{\mathrm{SD}, \text { coll }}^{\text {(unscaled) }}=1-\frac{(1-z) E_{R}+P_{L}}{(1-z) E_{R}+E_{L}}=\frac{E_{L}-P_{L}}{(1-z) E_{R}+E_{L}},
$$




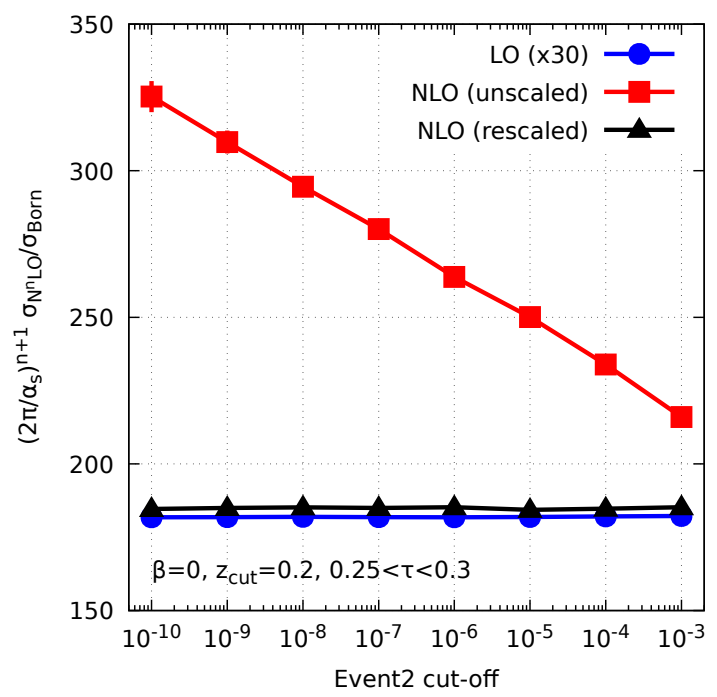

Figure 10. Illustration of the collinear divergence for unscaled soft-drop thrust using EVENT2 simulations.

which obviously differs from (A.1) by a finite amount independent of the angle of the collinear emission in the right hemisphere. For the corresponding virtual corrections (unscaled) soft-drop thrust would still be given by (A.1), yielding a mis-cancellation between real and virtual contributions and hence a collinear unsafety. It is worth noting that this collinear divergence starts at $\mathcal{O}\left(\alpha_{s}^{2}\right)$ since at $\mathcal{O}\left(\alpha_{s}\right)$ there is only one particle in the left hemisphere (the only emission being the collinear one) and $E_{L}=P_{L}$.

If instead one introduces the scaling factor of eq. (2.4), one gets

$$
\tau_{\mathrm{SD}}=\tau_{\mathrm{SD}, \text { coll }}=\frac{E_{L}-P_{L}}{Q},
$$

and collinear safety is restored.

To better illustrate the divergence, figure 10 shows the LO $\left(\mathcal{O}\left(\alpha_{s}\right)\right)$ and NLO $\left(\mathcal{O}\left(\alpha_{s}^{2}\right)\right)$ cross sections for soft-drop thrust, with $\beta=0$ and $z_{\text {cut }}=0.2$, to be between 0.25 and 0.3 as obtained using EVENT2 [35, 36]. The results are presented as a function of the soft cut-off used in the program. It is evident from the figure that the unscaled version of soft-drop thrust exhibits a logarithmic divergence as the cut-off is decreased while the rescaled version is collinear safe.

We note that this collinear unsafety issue is only present for $\beta=0$. Indeed, when $\beta>0$ the maximum value of $z$ depends on the angle of the emissions and goes to zero like a power of this angle. As a consequence, eq. (A.2) coincides with (A.1) in the collinear limit and the unsafety is absent.

\section{B Changes to the analytical calculation}

The analytical calculation we employ in this study does not deviate significantly from what was previously presented in ref. [19]. Therefore, in this appendix we limit ourselves 
to the presentation of those expressions we actually use and focus on any difference to the aforementioned paper, the notation of which we follow rather closely.

\section{B.1 Resummation equations}

The all-order expression for the soft-drop thrust distribution, in the region where the softdrop condition is active can be written as [19]:

$$
\Sigma(\tau)=\frac{1}{2 \pi i} \int_{C} \frac{d N}{N}\left[1+\sum_{n=1}^{\infty}\left(\frac{\alpha_{s}}{\pi}\right)^{n} \tilde{C}^{(n)}\right] e^{-R\left(\lambda_{\bar{N}}, \lambda_{z_{\text {cut }}}\right)},
$$

where $\tilde{C}$ encapsulates the constant contributions in $\tau$ and $z_{\text {cut }}$, which are neglected at NLL accuracy, and $\alpha_{s}$ is evaluated at scale $\mu$. The resummed exponent $R$ in the conjugate moment space is given by

$$
R\left(\lambda_{\bar{N}}, \lambda_{z_{\text {cut }}}\right)=-\frac{1}{\alpha_{s}} f_{1}\left(\lambda_{\bar{N}}, \lambda_{z_{\text {cut }}}\right)-f_{2}\left(\lambda_{\bar{N}}, \lambda_{z_{\text {cut }}}\right)-\alpha_{s} f_{3}\left(\lambda_{\bar{N}}, \lambda_{z_{\text {cut }}}\right)-\ldots
$$

with $\lambda_{\bar{N}}=\alpha_{s} b_{0} \log \left(x_{L} \bar{N}\right)$, where $\bar{N}=N e^{\gamma_{E}}$, and $\lambda_{z_{\text {cut }}}=\alpha_{s} b_{0} \log \left(x_{L} 2^{\beta / 2} z_{\text {cut }}\right)$. The parameter $x_{L}$ is an arbitrary rescaling factor that we vary in order to estimate missing higher-order corrections in the resummation, cf. section 5 . Note, the term $f_{3}$ does not contribute at NLL accuracy. The remaining functions $f_{i}(i=1,2)$ can be expressed through

$$
\begin{aligned}
f_{1}^{K}\left(\lambda_{T}\right)= & \frac{\Gamma_{K}^{(0)}}{2 b_{0}^{2} \pi}\left[\left(1+2 \lambda_{T}\right) \log \left(1+2 \lambda_{T}\right)-2 \lambda_{T}\right] \\
f_{2}^{K}\left(\lambda_{T}\right)= & \frac{\Gamma_{K}^{(1)}}{b_{0}^{2} \pi^{2}}\left[\lambda_{T}-\frac{1}{2} \log \left(1+2 \lambda_{T}\right)\right]+\frac{\Gamma_{K}^{(0)} b_{1}}{4 b_{0}^{3} \pi}\left[\log \left(1+2 \lambda_{T}\right)\left(2+\log \left(1+2 \lambda_{T}\right)\right)-4 \lambda_{T}\right] \\
& +\frac{\Gamma_{K}^{(0)}}{2 b_{0} \pi} \log \left(1+2 \lambda_{T}\right) L_{\mu}^{K}-\frac{\gamma_{K}^{(0)}}{2 b_{0} \pi} \log \left(1+2 \lambda_{T}\right)
\end{aligned}
$$

according to

$$
f_{i}(x, y)=f_{i}^{S_{G}}\left(p_{S_{G}}^{\left(z_{\mathrm{cut}}\right)} y\right)+2 f_{i}^{S_{C}}\left(p_{S_{C}}^{(\bar{N})} x+p_{S_{C}}^{\left(z_{\mathrm{cut}}\right)} y\right)+2 f_{i}^{J}\left(p_{J}^{(\bar{N})} x\right) .
$$

Here $S_{G}, S_{C}$ and $J$ indicate contributions from soft wide-angle, soft collinear and hard collinear regions, respectively. We here furthermore used the short-hand notation

$$
L_{\mu}^{K}=\log \left(\frac{Q^{2}}{\mu^{2}}\right)+2 p_{K}^{(2)} \log 2-\left(p_{K}^{\left(z_{\text {cut }}\right)}-p_{K}^{(\bar{N})}\right) \log x_{L} .
$$

The explicit coefficients needed for NLL accuracy read

$$
\begin{aligned}
\Gamma_{J} & =2 C_{F} \Gamma_{\text {cusp }}, \\
\Gamma_{S_{G}} & =\frac{2}{\beta+1} C_{F} \Gamma_{\text {cusp }}, \\
\Gamma_{S_{C}} & =-\frac{\beta+2}{\beta+1} C_{F} \Gamma_{\text {cusp }}, \\
\gamma_{J}^{(0)} & =\frac{3}{2} C_{F}, \\
\gamma_{S_{G}}^{(0)}=\gamma_{S_{C}}^{(0)} & =0
\end{aligned}
$$


where

$$
\Gamma_{\text {cusp }}=\sum_{n=0}^{\infty} \Gamma_{\text {cusp }}^{(n)}\left(\frac{\alpha_{s}}{\pi}\right)^{n+1}, \quad \text { with } \quad \Gamma_{\text {cusp }}^{(0)}=1, \Gamma_{\text {cusp }}^{(1)}=\frac{C_{A}}{2}\left(\frac{67}{18}-\frac{\pi^{2}}{6}\right)-\frac{5}{9} T_{R} n_{f} .
$$

Finally, the power coefficients are given by

$$
\begin{array}{ll}
p_{J}^{(\bar{N})}=-\frac{1}{2}, & p_{J}^{\left(z_{\text {cut }}\right)}=0 \\
p_{S_{G}}^{(\bar{N})}=0, & p_{S_{G}}^{\left(z_{\text {cut }}\right)}=1 \\
p_{S_{C}}^{(\bar{N})}=\frac{\beta+1}{\beta+2}, & p_{S_{C}}^{\left(z_{\text {cut }}\right)}=\frac{1}{\beta+2},
\end{array}
$$

and $p_{K}^{(2)}=0$ for all $K$.

\section{B.2 Treatment of the transition point}

A significant difference with respect to the approach of ref. [19] is the observation that, if we limit ourselves to NLL, as we do in this study, the transition point, marking the boundary between the groomed and ungroomed regions, can be taken at $\tau=z_{\text {cut }} 2^{\beta / 2}$. Thus, we naturally resum logarithms of $x_{L} z_{\text {cut }} 2^{\beta / 2}$ instead of logarithms of $x_{L} z_{\text {cut }} / 2$ and consequently all coefficients for the logarithms of 2 vanish, i.e. $p_{K}^{(2)}=0$ for all $K$.

We also include an additional correction associated with a discontinuity of the differential soft-drop thrust distribution at $\tau=z_{\beta} \equiv z_{\text {cut }} 2^{\beta / 2}$ at NLL accuracy, which was not included in ref. [19]. To identify the origin of the discontinuity, let us start with the expression for the resummed (cumulative) as given e.g. in ref. [37]:

$$
\Sigma_{\mathrm{res}}(\tau)=\lim _{\epsilon \rightarrow 0} \sum_{n=0}^{\infty} \frac{1}{n !} \prod_{i=1}^{n} \int_{\epsilon \tau}^{\tau} \frac{d \tau_{i}}{\tau_{i}} R^{\prime}\left(\tau_{i}\right) \exp [-R(\epsilon \tau)] \Theta\left(\tau-\sum_{i=1}^{n} \tau_{i}\right)
$$

where the exponent is related to the one presented in the previous subsection as $R(\tau)=$ $\left.R\left(\lambda_{\bar{N}}, \lambda_{z_{\text {cut }}}\right)\right|_{N \rightarrow \tau}$. One can then use the following expansions:

$$
\begin{aligned}
& R(\epsilon \tau)=R(\tau)+\log \left(\frac{1}{\varepsilon}\right) R^{\prime}(\tau)+\frac{1}{2} \log ^{2}\left(\frac{1}{\varepsilon}\right) R^{\prime \prime}(\tau)+\mathcal{O}\left(R^{\prime \prime \prime}(\tau)\right), \\
& R^{\prime}\left(\tau_{i}\right)=R^{\prime}(\tau)+\log \left(\frac{\tau}{\tau_{i}}\right) R^{\prime \prime}(\tau)+\mathcal{O}\left(R^{\prime \prime \prime}(\tau)\right) .
\end{aligned}
$$

At NLL accuracy, we can only keep the terms proportional to $R(\tau)$ and $R^{\prime}(\tau)$. Eg. (B.16) can then be evaluated and one gets

$$
\Sigma_{\mathrm{res}}^{(\mathrm{NLL})}(\tau)=\frac{\exp \left[-R(\tau)-\gamma_{E} R^{\prime}(\tau)\right]}{\Gamma\left(1+R^{\prime}(\tau)\right)} .
$$

For soft-drop thrust, the issue is that $R^{\prime \prime}(\tau)$ is discontinuous at $\tau=z_{\beta}$, meaning that if one computes the differential distribution by taking the derivative of (B.19), one would get a discontinuity at the transition point $\tau=z_{\beta}$. 
Since this contribution is proportional to $R^{\prime \prime}(\tau)$, it is formally NNLL in $\log (1 / \tau)$. Here, we want to extract from the NNLL corrections the part responsible for the discontinuity and include it as a transition-point correction. To do this, we now include the terms proportional to $R^{\prime \prime}(\tau)$ in (B.17). We first consider the case where $\tau<z_{\beta}$ for which (B.16) becomes

$$
\begin{aligned}
\left.\Sigma_{\mathrm{res}}(\tau)\right|_{\tau<z_{\beta}}= & \lim _{\epsilon \rightarrow 0} \sum_{n=0}^{\infty} \frac{1}{n !} \prod_{i=1}^{n} \int_{\epsilon \tau}^{\tau} \frac{d \tau_{i}}{\tau_{i}}\left[R^{\prime}(\tau)+\log \left(\frac{\tau}{\tau_{i}}\right) R^{\prime \prime}(\tau)\right] \\
& \times \exp \left[-R(\tau)-\log \left(\frac{1}{\epsilon}\right) R^{\prime}(\tau)-\frac{1}{2} \log ^{2}\left(\frac{1}{\epsilon}\right) R^{\prime \prime}(\tau)\right] \Theta\left(\tau-\sum_{i=1}^{n} \tau_{i}\right) .
\end{aligned}
$$

At the accuracy of interest, it is sufficient to include a single correction proportional to $R^{\prime \prime}(\tau)$ and one obtains after a few trivial manipulations

$$
\left.\Sigma_{\mathrm{res}}(\tau)\right|_{\tau<z_{\beta}}=\frac{\exp \left[-R(\tau)-\gamma_{E} R^{\prime}(\tau)\right]}{\Gamma\left(1+R^{\prime}(\tau)\right)}\left\{1+\int_{0}^{1} \frac{d x_{0}}{x_{0}} \log \left(\frac{1}{x_{0}}\right) R^{\prime \prime}(\tau)\left[\left(1-x_{0}\right)^{R^{\prime}(\tau)}-1\right]\right\} .
$$

The integration over $x_{0}$ in the above expression can be performed and one recovers the soft-collinear NNLL corrections associated with multiple-emission (cf. ref. [62]).

When $\tau>z_{\beta}$, one has to be a bit more careful as the expansions in (B.17) can involve crossing the transition point where $R^{\prime \prime}$ is discontinuous. In particular, for $\tau_{i}<z_{\beta}<\tau$ and for $\epsilon \tau<z_{\beta}<\tau$, one should instead use

$$
\begin{aligned}
\tau_{i}<z_{\beta}<\tau: \quad R^{\prime}\left(\tau_{i}\right)= & R^{\prime}(\tau)+\log \left(\frac{\tau}{z_{\beta}}\right) R^{\prime \prime}(\tau)+\log \left(\frac{z_{\beta}}{\tau_{i}}\right) R^{\prime \prime}\left(z_{\beta}\right)+\mathcal{O}\left(R^{\prime \prime \prime}(\tau)\right), \\
\epsilon \tau<z_{\beta}<\tau: \quad R(\epsilon \tau)= & R(\tau)+\log \left(\frac{1}{\epsilon}\right) R^{\prime}(\tau) \\
& +\frac{1}{2} \log ^{2}\left(\frac{\tau}{z_{\beta}}\right) R^{\prime \prime}(\tau)+\log \left(\frac{\tau}{z_{\beta}}\right) \log \left(\frac{z_{\beta}}{\epsilon \tau}\right) R^{\prime \prime}(\tau) \\
& +\frac{1}{2} \log ^{2}\left(\frac{z_{\beta}}{\epsilon \tau}\right) R^{\prime \prime}\left(z_{\beta}\right)+\mathcal{O}\left(R^{\prime \prime \prime}(\tau)\right) .
\end{aligned}
$$

Following the same procedure as for $\tau<z_{\beta}$ leads to

$$
\begin{aligned}
\left.\Sigma_{\mathrm{res}}(\tau)\right|_{\tau>z_{\beta}}= & \frac{\exp \left[-R(\tau)-\gamma_{E} R^{\prime}(\tau)\right]}{\Gamma\left(1+R^{\prime}(\tau)\right)}\left\{1+\int_{z_{\beta} / \tau}^{1} \frac{d x_{0}}{x_{0}} \log \left(\frac{1}{x_{0}}\right) R^{\prime \prime}(\tau)\left[\left(1-x_{0}\right)^{R^{\prime}(\tau)}-1\right]\right. \\
& +\int_{0}^{z_{\beta} / \tau} \frac{d x_{0}}{x_{0}}\left[\log \left(\frac{\tau}{z_{\beta}}\right)\left\{R^{\prime \prime}(\tau)-R^{\prime \prime}\left(z_{\beta}\right)\right\}+\log \left(\frac{1}{x_{0}}\right) R^{\prime \prime}\left(z_{\beta}\right)\right] \\
& \left.\times\left[\left(1-x_{0}\right)^{R^{\prime}(\tau)}-1\right]\right\} .
\end{aligned}
$$

One can further simplify these expressions by keeping only the contributions associated with the transition point. This is equivalent to subtracting the NNLL correction below the transition point, i.e. the correction that a standard NNLL calculation would give, from 
both (B.20) and (B.23). One then obtains our final expressions

$$
\begin{aligned}
\left.\Sigma_{\mathrm{res}}(\tau)\right|_{\tau<z_{\beta}}= & \frac{\exp \left[-R(\tau)-\gamma_{E} R^{\prime}(\tau)\right]}{\Gamma\left(1+R^{\prime}(\tau)\right)}, \\
\left.\Sigma_{\mathrm{res}}(\tau)\right|_{\tau>z_{\beta}}= & \frac{\exp \left[-R(\tau)-\gamma_{E} R^{\prime}(\tau)\right]}{\Gamma\left(1+R^{\prime}(\tau)\right)} \\
& \times\left\{1+\int_{0}^{z_{\beta} / \tau} \frac{d x_{0}}{x_{0}}\left[\log \left(\frac{\tau}{z_{\beta}}\right)\left[R^{\prime \prime}(\tau)-R^{\prime \prime}\left(z_{\beta}\right)\right]\right]\left[\left(1-x_{0}\right)^{R^{\prime}(\tau)}-1\right]\right\} \\
= & \frac{\exp \left[-R(\tau)-\gamma_{E} R^{\prime}(\tau)\right]}{\Gamma\left(1+R^{\prime}(\tau)\right)} \\
& \times \exp \left\{\int_{0}^{z_{\beta} / \tau} \frac{d x_{0}}{x_{0}}\left[\log \left(\frac{\tau}{z_{\beta}}\right)\left[R^{\prime \prime}(\tau)-R^{\prime \prime}\left(z_{\beta}\right)\right]\right]\left[\left(1-x_{0}\right)^{R^{\prime}(\tau)}-1\right]\right\}
\end{aligned}
$$

where the exponentiation of this contribution holds at NLL accuracy for both cumulative cross section and the differential distribution. Here the universal contribution is the usual Mellin inversion contribution, whereas an additional is included above the transition point.

One can show that the above result is, as expected, continuous at $\tau=z_{\beta}$. It is however interesting to comment on how this happens. The correction in the curly bracket in (B.24) are contributing only at the NNLL accuracy, and one can view the $\log \left(z_{\beta} / \tau\right)$ in the exponent as being small. However, when taking the derivative with respect to $\log (1 / \tau)$, it gives a correction which exactly compensates the divergence in (B.19). This extra dependence of the coefficient of $R^{\prime \prime}(\tau)$ contrasts with the standard case (cf. eq. (B.20)) where it does not depend on $\tau$ at NNLL accuracy.

\section{B.3 End-point correction}

Here we briefly describe our treatment to ensure that the resummed cross section and its expansion respect the kinematic end-point $\tau_{\max }$ of the fixed-order calculation, we thereby follow the procedure given in refs. [40, 41]. The primary modification is an alteration of the argument of the logarithm

$$
\log \left(x_{L} \tau\right) \rightarrow-\frac{1}{p} \log \left(\frac{1}{\left(x_{L} \tau\right)^{p}}-\frac{1}{\left(x_{L} \tau_{\max }\right)^{p}}+1\right)=\log \bar{\tau}
$$

where the parameter $p$ determines the slope of the approach to the end-point.

However, the modification of the logarithm is not enough in order to ensure for the derivative of the expansion to approach 0 . To implement this an additional contribution is included in the resummed exponential

$$
\Sigma_{\text {res }} \rightarrow \Sigma_{\text {res }} \exp \left[-\left(\frac{\tau}{\tau_{\max }}\right)^{p} \tilde{R}^{\prime}\left(\tau \rightarrow \tau_{\max }\right) \log \bar{\tau}\right]
$$

where $\tilde{R}$ includes the transition-point and multiple-emission effects. The derivative here is taken with respect to $\log \bar{\tau}$.

The value of the end-point depends on the accuracy of the fixed-order calculation which we match to. At NLO precision this is given by $\tau_{\max }=0.4225$ [41]. 


\section{An analytic model for hadronisation corrections}

In this section the analytic hadronisation model is described in detail. The derivation follows the approach of [60], which was applied to mMDT and soft drop in [30,61].

\section{C.1 Non-perturbative effects on plain thrust}

For plain thrust non-perturbative corrections result in a simple shift of the observable value. To derive this shift we evaluate the observable for a $2 \rightarrow 2$ process with one additional nonperturbative emission $k$ off the hard legs 1 and 2. We find it convenient to align the $z$ axis with one of the final-state particles, so the kinematics for this computation are given by

$$
\begin{aligned}
p_{2} & =E_{2}(1,0,0,1), \\
k & =E_{k}\left(1,0, \sqrt{1-c_{2 k}^{2}}, c_{2 k}\right),
\end{aligned}
$$

where $c_{2 k}=\cos \theta_{2 k}$, with $\theta_{23}$ the angle between leg 2 and the soft non-perturbative emission $k$, while $p_{1}=q-p_{2}-k$, with $q=Q(1,0,0,0)$. Exploiting energy momentum conservation and on-shell relations, we can express the energies $E_{1}$ and $E_{2}$ as a function of the energy of the non-perturbative emission $E_{k}$ and $c_{2 k}$

$$
\begin{aligned}
& E_{1}=\frac{Q^{2}-2\left(1-c_{2 k}\right) E_{k}\left(Q-E_{k}\right)}{2\left(Q-E_{k}\left(1-c_{2 k}\right)\right)}, \\
& E_{2}=\frac{Q\left(Q-2 E_{k}\right)}{2\left(Q-E_{k}\left(1-c_{2 k}\right)\right)} .
\end{aligned}
$$

This results in a contribution to the thrust

$$
\begin{array}{ll}
\tau(k)=\delta \tau^{+}(k)=1-\frac{2 E_{1}}{Q}=\frac{E_{k}\left(Q-2 E_{k}\right)\left(1-c_{2 k}\right)}{Q\left(Q-E_{k}\left(1-c_{2 k}\right)\right)}, & \text { when } c_{2 k}>-\frac{E_{k}}{Q-E_{k}}, \\
\tau(k)=\delta \tau^{-}(k)=1-\frac{2 E_{2}}{Q}=\frac{E_{k}\left(1+c_{2 k}\right)}{\left(Q-E_{k}\left(1-c_{2 k}\right)\right)}, & \text { when } c_{2 k}<-\frac{E_{k}}{Q-E_{k}},
\end{array}
$$

where the two cases correspond to the hemisphere in which the non-perturbative emission resides. Making use of the eikonal rules we can write down an integral for the expectation value of the shift as a result of this non-perturbative emission:

$$
\langle\delta \tau\rangle_{h}=C_{F} \int d E_{k} E_{k} d c_{2 k} \frac{\alpha_{s}\left(k_{t, 12}\right)}{2 \pi} \frac{p_{1} \cdot p_{2}}{\left(p_{1} \cdot k\right)\left(p_{2} \cdot k\right)} \delta \tau(k),
$$

where the scale for $\alpha_{s}[63]$ is given by

$$
k_{t, 12}^{2}=2 \frac{\left(p_{1} \cdot k\right)\left(p_{2} \cdot k\right)}{p_{1} \cdot p_{2}}
$$

and

$$
\begin{aligned}
\delta \tau(k) & =\delta \tau^{+}(k) \Theta\left(c_{2 k}+\frac{E_{k}}{Q-E_{k}}\right)+\delta \tau^{-}(k)\left[1-\Theta\left(c_{2 k}+\frac{E_{k}}{Q-E_{k}}\right)\right] \\
& =\delta \tau^{-}(k)+\Theta\left(c_{2 k}+\frac{E_{k}}{Q-E_{k}}\right)\left[\delta \tau^{+}(k)-\delta \tau^{-}(k)\right] .
\end{aligned}
$$


It can be shown [60] that the first contribution is power-suppressed with the exception of the collinear divergence, which will anyway cancel. Therefore we shall focus on the difference

$$
\delta \tau^{+}(k)-\delta \tau^{-}(k)=-\frac{2 E_{k}}{Q} \frac{Q c_{2 k}+E_{k}\left(1-c_{2 k}\right)}{Q-E_{k}\left(1-c_{2 k}\right)} .
$$

In addition, with $k_{t, 12}$ being the argument of $\alpha_{s}$, we change the integration variable to

$$
k_{t, 12}=E_{k} \sqrt{1-c_{2 k}^{2}} \frac{Q}{Q-E_{k}\left(1-c_{2 k}\right)},
$$

which, for simplicity, we denote as $k_{t}$ in what follows. This results in the final integral

$$
\langle\delta \tau\rangle_{h}=-C_{F} \int_{0}^{\mu_{I}} \frac{d k_{t}}{Q} \frac{\delta \alpha_{s}\left(k_{t}\right)}{\pi} \int_{-1}^{1} d c_{2 k}\left[2 c_{2 k}\left(1-c_{2 k}^{2}\right)^{-3 / 2} \Theta\left(c_{2 k}\right)+\mathcal{O}\left(k_{t} / Q\right)\right],
$$

where we have performed an expansion with $k_{t}$ as the softness parameter. $\delta \alpha_{s}\left(k_{t}\right)$ denotes the difference between the non-perturbative and the perturbative description of the strong coupling. The angular integral can easily be performed, with the collinear divergence cancelling against the first term in (C.8), and we can redefine the $k_{t}$ integral

$$
\langle\delta \tau\rangle_{h}=2 C_{F} \frac{\Lambda\left(\mu_{I}\right)}{Q}
$$

where we have introduced

$$
\Lambda\left(\mu_{I}\right)=\int_{0}^{\mu_{I}} \frac{d k_{t}}{\pi} \delta \alpha_{s}\left(k_{t}\right) .
$$

The actual fitted parameter is defined as

$$
\Omega=C_{F} \Lambda\left(\mu_{I}\right) .
$$

\section{C.2 Non-perturbative effects on soft-drop thrust}

Now that we have reviewed the effect of a non-perturbative emission on the value of thrust we can study the groomed distribution. In general a non-perturbative emission for the $2 \rightarrow 2$ case will be groomed away leaving no change in thrust unless the emission is hard enough and very collinear (resulting in a small $k_{t}$ but large enough energy).

This can be computed by including the soft-drop restriction for the non-perturbative emission:

$$
k_{t}>z_{\text {cut }} \frac{Q}{2}\left(1-c_{2 k}\right)^{(1+\beta) / 2}\left(1+c_{2 k}\right)^{1 / 2},
$$

where the small $k_{t}$ approximation has been used. This can be rewritten as a condition on $c_{2 k}$ under the assumption that $1+c_{2 k}$ is close to 2 , i.e.

$$
c_{2 k}>1-\left(\frac{\sqrt{2} k_{t}}{z_{\text {cut }} Q}\right)^{2 /(\beta+1)} .
$$

The integral will now be over $\delta \tau^{+}(k)$ instead of the difference as there is no shift if the emission is groomed. Performing the angular integral results in a contribution:

$$
\langle\delta \tau\rangle_{h, \mathrm{SD}}=2^{(\beta+2) /(2(\beta+1))} C_{F} z_{\text {cut }}^{-1 /(\beta+1)} \frac{\Lambda_{\mathrm{SD}}\left(\mu_{I}, \beta\right)}{Q^{(\beta+2) /(\beta+1)}},
$$


including a factor 2 for both hemispheres where

$$
\Lambda_{\mathrm{SD}}\left(\mu_{I}, \beta\right)=\int_{0}^{\mu_{I}} \frac{k_{t}^{1 /(\beta+1)} d k_{t}}{\pi} \delta \alpha_{s}\left(k_{t}\right) .
$$

This effect is suppressed in $k_{t}$. Note that the limit $\beta \rightarrow \infty$ does not work in this case as there are contributions of the type $2-\left(\sqrt{2} k_{t} /\left(z_{\text {cut }} Q\right)\right)^{2 /(\beta+1)}$, which approach 1 for $\beta \rightarrow \infty$ but result in a factor 2 in the small $k_{t}$ limit.

A contribution which is not suppressed originates from a $2 \rightarrow n$ process where the non-perturbative emission is protected by larger-angle hard emissions, which pass soft drop. This is given by:

$$
\langle\delta \tau\rangle_{h}=C_{F} \int d E_{k} E_{k} d c_{23, k} \frac{d \phi_{k}}{2 \pi} \frac{\alpha_{s}\left(k_{t, i j}\right)}{2 \pi} \frac{p_{1} \cdot p_{23}}{\left(p_{1} \cdot k\right)\left(p_{23} \cdot k\right)} \delta \tau^{+}(k) \Theta\left(c_{23, k}-c_{23}\right),
$$

where we apply the same shift as for the $2 \rightarrow 2$ kinematics with an angular constraint now set by the angle $\theta_{23}$ between the emissions passing the soft-drop condition. Here, the non-perturbative emission is constrained by the angle between the two hard particles in one hemisphere, $c_{23, k}>c_{23}$. This condition can be translated to a restriction based on a value of thrust. Here we make use of the same kinematics as in last subsection with $k \rightarrow p_{3}$, thus

$$
\tau=\frac{E_{2}\left(Q-2 E_{2}\right)\left(1-c_{23}\right)}{Q\left(Q-E_{2}\left(1-c_{23}\right)\right)}
$$

resulting in

$$
c_{23}=1-\frac{2 \tau}{(1-z)(z+\tau)},
$$

with $z=1-2 E_{2} / Q$.

We have once again

$$
k_{t, 1,23}=E_{k} \sqrt{1-c_{23, k}^{2}} \frac{Q}{Q-E_{k}\left(1-c_{23, k}\right)} .
$$

This results in the integral

$$
\begin{aligned}
\langle\delta \tau\rangle_{h} & =C_{F} \int \frac{d k_{t}}{Q} \frac{\delta \alpha_{s}\left(k_{t}\right)}{2 \pi} d x \frac{d \phi_{k}}{2 \pi} \frac{\tau^{1 / 2}(1-z)(\tau+z)}{x^{1 / 2}(\tau(1-x-z)+(1-z) z)^{3 / 2}}+\mathcal{O}\left(k_{t} / Q\right) \\
& =C_{F} \frac{\Lambda\left(\mu_{I}\right)}{Q} \frac{\tau^{1 / 2}}{(z(1-z-\tau))^{1 / 2}}=C_{F} \frac{\Lambda\left(\mu_{I}\right)}{Q} \frac{\tau^{1 / 2}}{(z(1-z))^{1 / 2}}+\mathcal{O}(\tau),
\end{aligned}
$$

with $1-c_{23, k}=x\left(1-c_{23}\right)$.

The mean value of this shift can be obtained by averaging the above result with the appropriate QCD splitting function. Here the integration boundaries will have a transition point in thrust:

$$
\left\langle(z(1-z))^{-1 / 2}\right\rangle=\frac{\int_{Z_{\mathrm{SD}}-\tau}^{1-Z_{\mathrm{SD}}} d z(z(1-z))^{-1 / 2} P_{q g}(z)}{\int_{Z_{\mathrm{SD}}-\tau}^{1-Z_{\mathrm{SD}}} d z P_{q g}(z)},
$$


for $\tau<z_{\text {cut }} / 2$, and

$$
\left\langle(z(1-z))^{-1 / 2}\right\rangle=\frac{\int_{\tau}^{1-2 \tau} d z(z(1-z))^{-1 / 2} P_{q g}(z)}{\int_{\tau}^{1-2 \tau} d z P_{q g}(z)},
$$

for $\tau>z_{\text {cut }} / 2$ with $Z_{\mathrm{SD}}=z_{\text {cut }}^{2 /(2+\beta)}(2 \tau)^{\beta /(2+\beta)}$. Finally for $\tau>1 / 3$ both these integrals approach 0 simultaneously and we make use of the limit

$$
\left\langle(z(1-z))^{-1 / 2}\right\rangle=\frac{3}{\sqrt{2}},
$$

however this is not relevant to the fitting range.

\section{C.3 Shift in energy}

In addition to shifting the value of thrust it is also possible to shift the energy of an emitted gluon downwards to below the needed energy fraction in order to pass the grooming condition. Effectively this shift in the energy can be interpreted as a shift in $z_{\text {cut }}$

$$
\delta z_{\text {cut }}=-2\left(\frac{(z+\tau)(1-z)}{2 \tau}\right)^{\beta / 2} \frac{\delta E}{Q},
$$

where we have made use of $E_{2}=(1-z) Q / 2$ and $E_{3}=(z+\tau) Q / 2$ and we have assumed the angle is fixed and determined by the value of thrust. Here we can compute the energy difference using the same techniques than those applied to derive the shift in thrust. Once again we will make use of the observable difference for the $2 \rightarrow 2$ case:

$$
\begin{aligned}
& \delta E^{+}(k)=E_{3}+E_{k}-Q / 2=\frac{E_{k}\left(Q-2 E_{k}\right)\left(1-c_{3 k}\right)}{2\left(Q-E_{k}\left(1-c_{3 k}\right)\right)}, \\
& \delta E^{-}(k)=E_{3}-Q / 2=\frac{-E_{k} Q\left(1+c_{3 k}\right)}{2\left(Q-E_{k}\left(1-c_{3 k}\right)\right)} .
\end{aligned}
$$

The first shift corresponds to the case where the particles $k$ and 3 are clustered together first, whereas the second case assumes $k$ does not cluster with 3 . This results in the integral

$$
\langle\delta E\rangle_{h}=C_{A} \int d E_{k} E_{k} d c_{2 k} \frac{\alpha_{s}\left(k_{t, 12}\right)}{2 \pi} \frac{p_{1} \cdot p_{3}}{\left(p_{1} \cdot k\right)\left(p_{3} \cdot k\right)}\left(\delta E^{+}-\delta E^{-}\right) \Theta\left(c_{3 k}-c_{23}\right) .
$$

Note the different colour factor with respect to the previous effect: we now expect the dominant contribution to come from a non-perturbative gluon that shifts the energy of the perturbative one. Again we use

$$
k_{t, 13}=E_{k} \sqrt{1-c_{3 k}^{2}} \frac{Q}{Q-E_{k}\left(1-c_{3 k}\right)} .
$$

Resulting in the integral

$$
\begin{aligned}
\langle\delta E\rangle_{h} & =C_{A} \int d k_{t} \frac{\delta \alpha_{s}\left(k_{t}\right)}{2 \pi} d c_{3 k} \frac{d \phi_{k}}{2 \pi} \frac{2}{\left(1-c_{3 k}^{2}\right)^{3 / 2}}+\mathcal{O}\left(k_{t} / Q\right) \\
& =-C_{A} \Lambda\left(\mu_{I}\right) \frac{c_{23}}{\sqrt{1-c_{23}^{2}}}=-C_{A} \Lambda\left(\mu_{I}\right) \frac{z(1-z-\tau)-\tau}{2 \sqrt{\tau z(1-z-\tau)}} .
\end{aligned}
$$


Since we know $z \sim Z_{\mathrm{SD}}-\tau$ we can fill this into the expression resulting in

$$
\left\langle\delta z_{\mathrm{cut}}\right\rangle_{h}=2 C_{A}\left(\frac{Z_{\mathrm{SD}}\left(1-Z_{\mathrm{SD}}+\tau\right)}{2 \tau}\right)^{\beta / 2} \frac{\left(Z_{\mathrm{SD}}-\tau\right)\left(1-Z_{\mathrm{SD}}\right)-\tau}{2 \sqrt{\tau\left(Z_{\mathrm{SD}}-\tau\right)\left(1-Z_{\mathrm{SD}}\right)}} \frac{\Lambda\left(\mu_{I}\right)}{Q} .
$$

However this only holds for $\tau<z_{\text {cut }} / 2$, therefore we freeze the shift for $\tau>z_{\text {cut }} / 2$ leading to

$$
\left\langle\delta z_{\text {cut }}\right\rangle_{h}=-2 C_{A}\left(1-z_{\text {cut }} / 2\right)^{\beta / 2} \frac{z_{\text {cut }}}{2 \sqrt{1-z_{\text {cut }}}} \frac{\Lambda\left(\mu_{I}\right)}{Q}, \quad \text { when } \tau>\frac{z_{\text {cut }}}{2} .
$$

\section{Validation of Hadronisation corrections from Monte Carlo generators}

In figure 11 we compare the predictions of various general purpose Monte Carlo generators with different hadronisation models for the various choices of $z_{\text {cut }}$ and $\beta$. Being mainly interested in modifications due to different fragmentation models, we perform this comparison for $\mathrm{LO} e^{+} e^{-} \rightarrow 2 j$ matrix elements with parton showers attached as the perturbative input for all generators. In addition to the Sherpa setups described in the main text, we use Sherpa with the Dire parton cascade [56] thus changing the underlying showering algorithm but still using cluster hadronisation model. We find relatively mild changes in the predicted hadronisation corrections with respect to using the dipole shower.

We furthermore compare to Herwig version 7.1.4 [55, 64] and Pythia version 8.235 [49, 54]. For Herwig we use the angular-ordered parton shower in conjunction with its cluster fragmentation model [65]. Pythia implements a transverse momentum ordered parton shower supplemented with the Lund fragmentation model. Both hadronisation models are used with their respective default tuning parameters [66-68]. The effect of soft-drop grooming on the thrust distribution is modelled consistently between the generators. We observe that the hadronisation corrections, taken from ratios between the nominal predictions of the generators to their respective parton level, are at most about $10 \%$ in the relevant range for most soft-drop parameters, irrespective of the generator used. For all but the most extreme choice of $z_{\text {cut }}=0.33$, the range between the default Sherpa dipole shower with cluster or string hadronisation cover the spread of the other generator choices, at least in the default fitting range of the observable. This justifies the exclusive use of the Sherpa dipole shower to generate pseudo data for the $\alpha_{s}$ fits and to determine hadronisation corrections, with the average of cluster and string model as our default choice and the difference to cluster fragmentation and the string model as an estimate of the related uncertainty.

An alternative way to account for hadronisation corrections other than the ratio between parton and hadron level is to obtain a bin-by-bin transition matrix from the Monte Carlo and apply it to the analytic calculation. We compare the two methods in figure 12, for plain thrust and for soft-drop thrust with parameters $z_{\text {cut }}=0.2$ and $\beta=0$ (other parameter choices show a similar behaviour). Although the methods are clearly not equivalent in the peak region and in the far tail, we find only small numerical differences in the region where the fit is performed, with almost no dependence on $\alpha_{s}$. Similarly, small effects are observed for other parameter choices. We conclude that the difference between these two methods does not significantly add to the uncertainty assigned to the hadronisation corrections, and is irrelevant at the overall level of accuracy considered. 


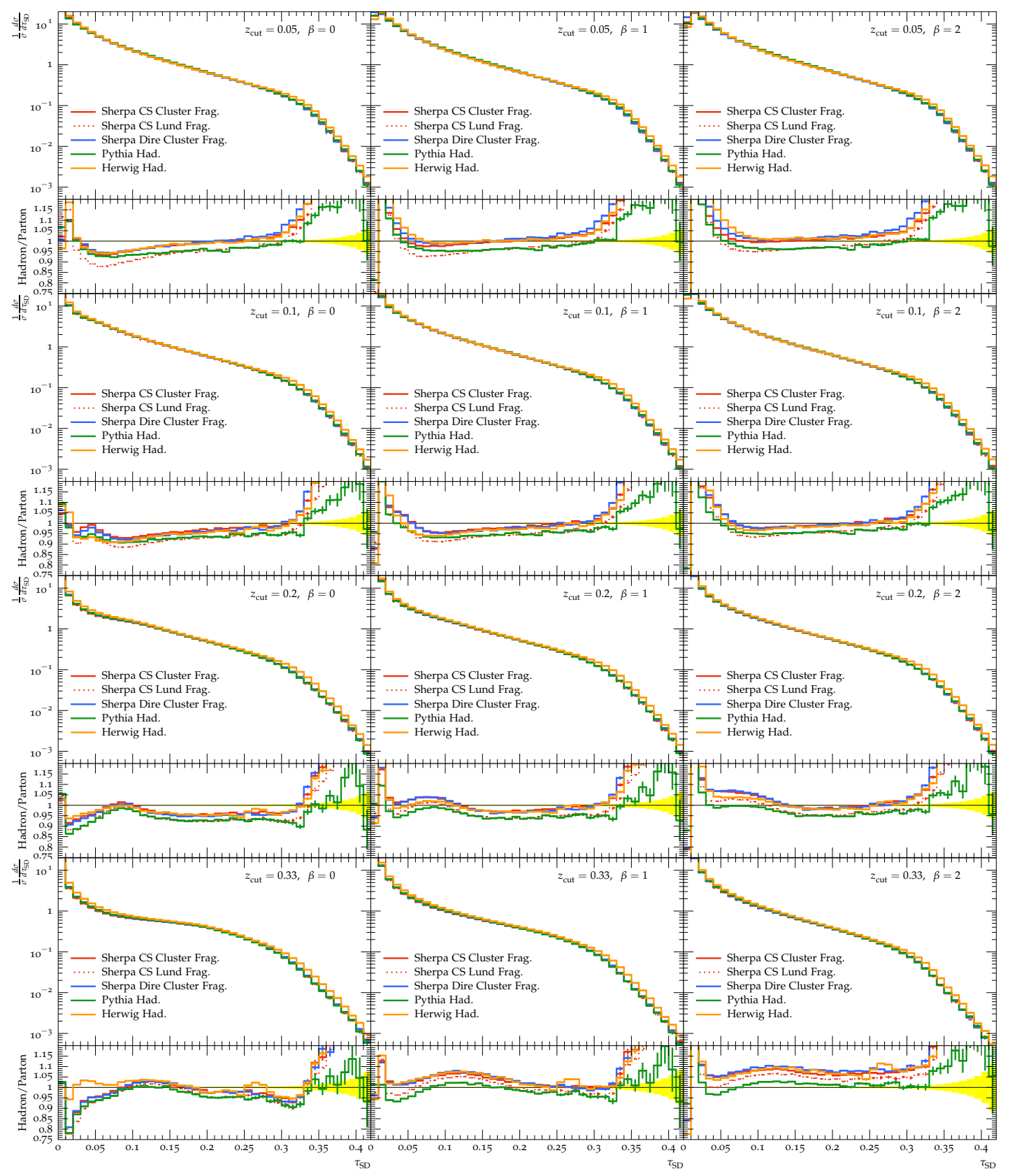

Figure 11. Predictions from general-purpose Monte Carlo generators for soft drop thrust with various $z_{\text {cut }}$ and $\beta$ values at parton shower accuracy. Shown are the nominal distributions at hadron level and the ratios of hadron level to the respective underlying parton level predictions. 

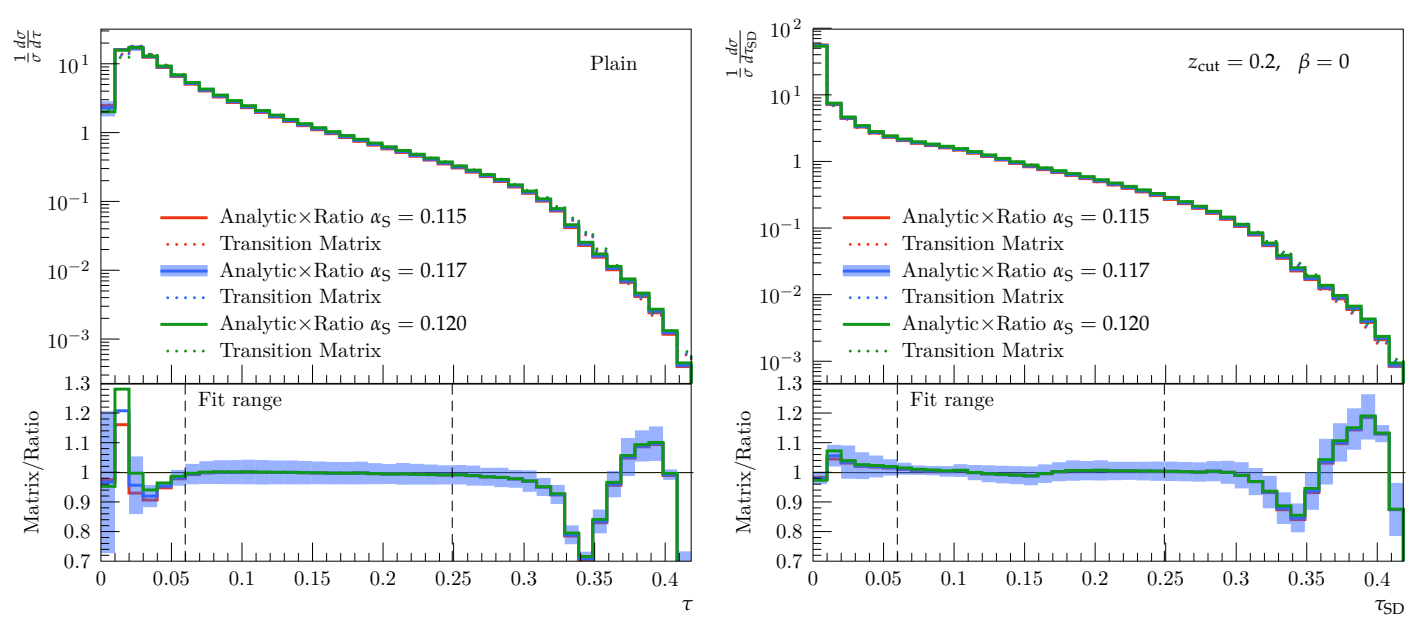

Figure 12. Comparison of different methods to apply hadronisation corrections obtained from Monte Carlo to the analytic calculations, for plain thrust (left) and soft-drop thrust (right) with $z_{\text {cut }}=0.2, \beta=0$. The corrections are obtained from simulations at parton shower accuracy and are applied either by multiplying the analytic calculation at various $\alpha_{s}$ values by the ratio from Monte Carlo, as described in the main text (solid) or alternatively by applying a bin-by-bin transition matrix obtained from the same Monte Carlo run (dotted). The cluster hadronisation model is used in all cases, the blue band indicates the uncertainty assigned to the calculation for $\alpha_{s}=0.117$ based on the difference to using the Lund model. The bottom panel shows the ratio between the distributions using the two methods for equal values of $\alpha_{s}$.

\section{E Alternative treatment of the resummation uncertainty}

In the study we have presented here we have chosen to estimate the theoretical uncertainty due to missing higher-logarithmic contributions in our resummation by rescaling the arguments of both the logarithms of thrust and of $z_{\text {cut }}$ by an arbitrary factor $x_{L}$, which we are free to vary. As an alternative, we can consider to include the rescaling factor in logarithms of thrust only. In this case the location of the transition point depends on the value of $x_{L}$. In order to test the impact of this another code was developed for the resummation which is formally equivalent at NLL accuracy, but uses the alternative treatment for estimating the resummation uncertainty. In figure 13 the results of the fit using the alternative approach can be seen. Here the central values are slightly different due to some formally NNLL differences. However, the main difference is the significantly reduced uncertainty. In fact the uncertainty shown here is smaller than for plain thrust. We have decided to adopt a rather conservative approach, i.e. using the method described in the main text and in appendix $\mathrm{B}$ as the default choice to estimate the resummation contribution to the total theoretical uncertainty.

Open Access. This article is distributed under the terms of the Creative Commons Attribution License (CC-BY 4.0), which permits any use, distribution and reproduction in any medium, provided the original author(s) and source are credited. 

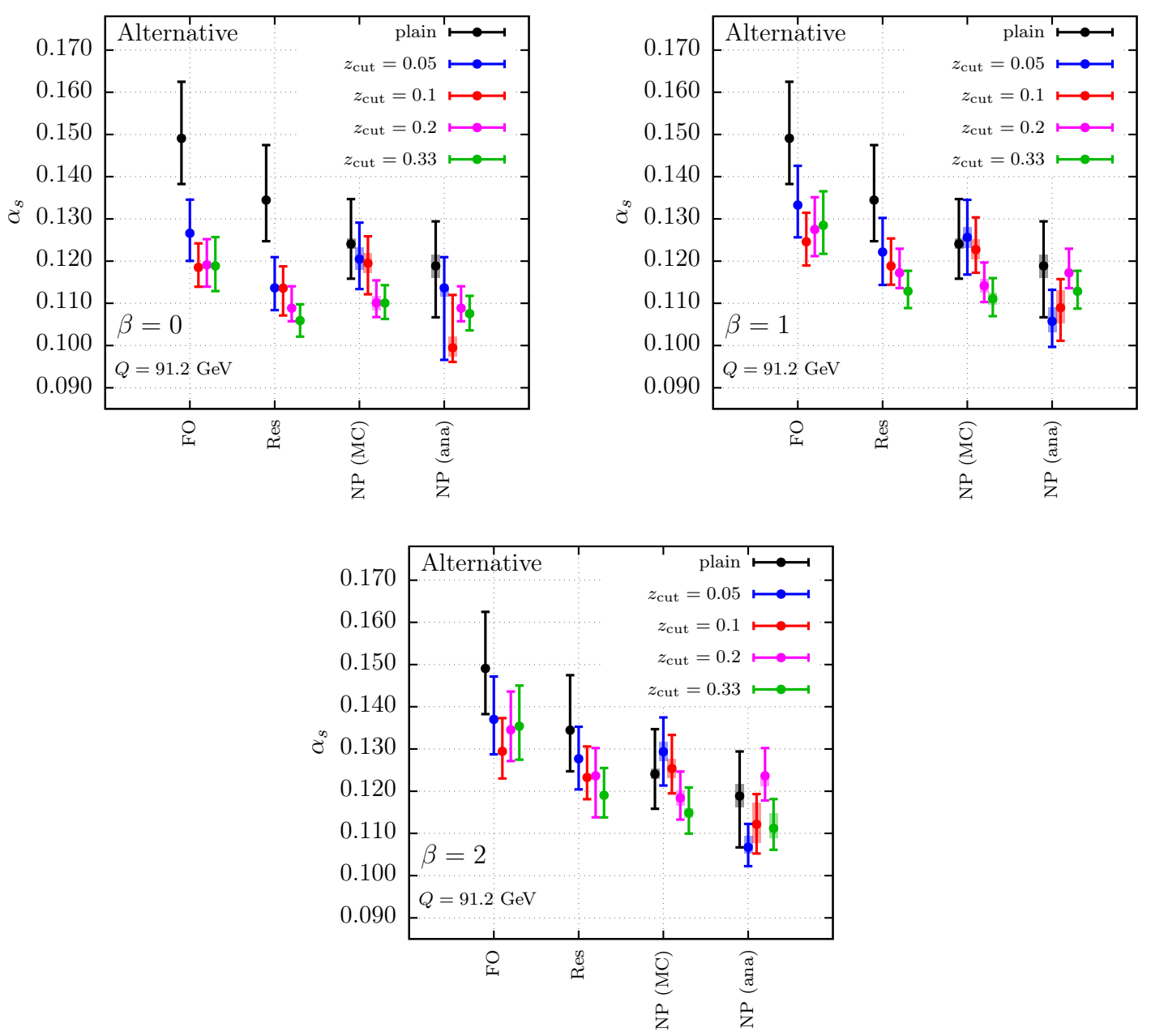

Figure 13. Similar results to figure 6 making use of an alternative resummation code with a different treatment for the resummation uncertainty.

\section{References}

[1] Particle Data Group collaboration, Review of particle physics, Phys. Rev. D 98 (2018) 030001 [INSPIRE].

[2] K. Maltman and T. Yavin, $\alpha_{s}\left(M_{Z}^{2}\right)$ from hadronic $\tau$ decays, Phys. Rev. D 78 (2008) 094020 [arXiv:0807.0650] [INSPIRE].

[3] PACS-CS collaboration, Precise determination of the strong coupling constant in $N_{f}=2+1$ lattice QCD with the Schrödinger functional scheme, JHEP 10 (2009) 053 [arXiv:0906. 3906] [INSPIRE].

[4] C. McNeile et al., High-precision $c$ and $b$ masses and QCD coupling from current-current correlators in lattice and continuum QCD, Phys. Rev. D 82 (2010) 034512 [arXiv: 1004.4285] [INSPIRE].

[5] B. Blossier et al., The strong running coupling at $\tau$ and $Z_{0}$ mass scales from lattice $Q C D$, Phys. Rev. Lett. 108 (2012) 262002 [arXiv:1201.5770] [INSPIRE]. 
[6] B. Chakraborty et al., High-precision quark masses and QCD coupling from $n_{f}=4$ lattice QCD, Phys. Rev. D 91 (2015) 054508 [arXiv: 1408.4169] [inSPIRE].

[7] Fermilab Lattice, MiLC collaboration, Charmed and light pseudoscalar meson decay constants from four-flavor lattice QCD with physical light quarks, Phys. Rev. D 90 (2014) 074509 [arXiv: 1407.3772] [inSPIRE].

[8] JADE collaboration, Determination of the strong coupling $\alpha_{s}$ from hadronic event shapes with $O\left(\alpha_{s}^{3}\right)$ and resummed QCD predictions using JADE data, Eur. Phys. J. C 64 (2009) 351 [arXiv:0810.1389] [INSPIRE].

[9] G. Dissertori et al., Determination of the strong coupling constant using matched $N N L O+N L L A$ predictions for hadronic event shapes in $e^{+} e^{-}$annihilations, JHEP 08 (2009) 036 [arXiv:0906.3436] [INSPIRE].

[10] G. Dissertori et al., Precise determination of the strong coupling constant at NNLO in QCD from the three-jet rate in electron-positron annihilation at LEP, Phys. Rev. Lett. 104 (2010) 072002 [arXiv: 0910.4283] [INSPIRE].

[11] OPAL collaboration, Determination of $\alpha_{s}$ using OPAL hadronic event shapes at $\sqrt{s}=91-209 \mathrm{GeV}$ and resummed NNLO calculations, Eur. Phys. J. C 71 (2011) 1733 [arXiv:1101.1470] [INSPIRE].

[12] JADE collaboration, Measurement of the strong coupling $\alpha_{s}$ from the three-jet rate in $e^{+} e^{-}$-annihilation using JADE data, Eur. Phys. J. C 73 (2013) 2332 [arXiv:1205.3714] [INSPIRE].

[13] R.A. Davison and B.R. Webber, Non-perturbative contribution to the thrust distribution in $e^{+} e^{-}$annihilation, Eur. Phys. J. C 59 (2009) 13 [arXiv:0809.3326] [INSPIRE].

[14] R. Abbate et al., Thrust at $N^{3} L L$ with power corrections and a precision global fit for $\alpha_{s}\left(m_{Z}\right)$, Phys. Rev. D 83 (2011) 074021 [arXiv: 1006.3080] [InSPIRE].

[15] T. Gehrmann, G. Luisoni and P.F. Monni, Power corrections in the dispersive model for a determination of the strong coupling constant from the thrust distribution, Eur. Phys. J. C 73 (2013) 2265 [arXiv:1210.6945] [INSPIRE].

[16] A.H. Hoang, D.W. Kolodrubetz, V. Mateu and I.W. Stewart, C-parameter distribution at $N^{3} L L^{\prime}$ including power corrections, Phys. Rev. D 91 (2015) 094017 [arXiv:1411.6633] [INSPIRE].

[17] E. Farhi, A QCD test for jets, Phys. Rev. Lett. 39 (1977) 1587 [InSPIRE].

[18] R. Abbate et al., Precision thrust cumulant moments at $N^{3} L L$, Phys. Rev. D 86 (2012) 094002 [arXiv: 1204.5746] [INSPIRE].

[19] J. Baron, S. Marzani and V. Theeuwes, Soft-drop thrust, JHEP 08 (2018) 105 [arXiv: 1803.04719] [INSPIRE].

[20] J.R. Andersen et al., Les Houches 2017: physics at TeV colliders standard model working group report, arXiv: 1803.07977.

[21] S. Marzani, G. Soyez and M. Spannowsky, Looking inside jets: an introduction to jet substructure and boosted-object phenomenology, arXiv:1901.10342 [INSPIRE].

[22] A.J. Larkoski, I. Moult and B. Nachman, Jet substructure at the Large Hadron Collider: a review of recent advances in theory and machine learning, arXiv:1709.04464 [INSPIRE]. 
[23] R. Kogler et al., Jet substructure at the Large Hadron Collider: experimental review, arXiv:1803.06991 [INSPIRE].

[24] A.J. Larkoski, S. Marzani, G. Soyez and J. Thaler, Soft drop, JHEP 05 (2014) 146 [arXiv: 1402.2657] [INSPIRE].

[25] E. Bothmann et al., Event generation with Sherpa 2.2, SciPost Phys. 7 (2019) 034 [arXiv: 1905.09127] [INSPIRE].

[26] T. Gleisberg et al., Event generation with SHERPA 1.1, JHEP 02 (2009) 007 [arXiv: 0811.4622] [INSPIRE].

[27] Y.L. Dokshitzer, G.D. Leder, S. Moretti and B.R. Webber, Better jet clustering algorithms, JHEP 08 (1997) 001 [hep-ph/9707323] [INSPIRE].

[28] M. Wobisch and T. Wengler, Hadronization corrections to jet cross-sections in deep inelastic scattering, hep-ph/9907280 [INSPIRE].

[29] J.M. Butterworth, A.R. Davison, M. Rubin and G.P. Salam, Jet substructure as a new Higgs search channel at the LHC, Phys. Rev. Lett. 100 (2008) 242001 [arXiv:0802.2470] [INSPIRE].

[30] M. Dasgupta, A. Fregoso, S. Marzani and G.P. Salam, Towards an understanding of jet substructure, JHEP 09 (2013) 029 [arXiv: 1307.0007] [INSPIRE].

[31] M. Cacciari, G.P. Salam and G. Soyez, FastJet user manual, Eur. Phys. J. C 72 (2012) 1896 [arXiv:1111.6097] [INSPIRE].

[32] C. Frye, A.J. Larkoski, M.D. Schwartz and K. Yan, Factorization for groomed jet substructure beyond the next-to-leading logarithm, JHEP 07 (2016) 064 [arXiv:1603.09338] [INSPIRE].

[33] A. Kardos, G. Somogyi and Z. Trócsányi, Soft-drop event shapes in electron-positron annihilation at next-to-next-to-leading order accuracy, Phys. Lett. B 786 (2018) 313 [arXiv: 1807.11472] [INSPIRE].

[34] S. Marzani, L. Schunk and G. Soyez, A study of jet mass distributions with grooming, JHEP 07 (2017) 132 [arXiv: 1704.02210] [INSPIRE].

[35] S. Catani and M.H. Seymour, The dipole formalism for the calculation of QCD jet cross-sections at next-to-leading order, Phys. Lett. B 378 (1996) 287 [hep-ph/9602277] [INSPIRE].

[36] S. Catani and M.H. Seymour, A general algorithm for calculating jet cross-sections in NLO QCD, Nucl. Phys. B 485 (1997) 291 [Erratum ibid. B 510 (1998) 503] [hep-ph/9605323] [INSPIRE].

[37] A. Banfi, G.P. Salam and G. Zanderighi, Principles of general final-state resummation and automated implementation, JHEP 03 (2005) 073 [hep-ph/0407286] [INSPIRE].

[38] E. Gerwick, S. Hoeche, S. Marzani and S. Schumann, Soft evolution of multi-jet final states, JHEP 02 (2015) 106 [arXiv: 1411.7325] [INSPIRE].

[39] A. Banfi, G.P. Salam and G. Zanderighi, Phenomenology of event shapes at hadron colliders, JHEP 06 (2010) 038 [arXiv: 1001.4082] [InSPIRE].

[40] S. Catani, L. Trentadue, G. Turnock and B.R. Webber, Resummation of large logarithms in $e^{+} e^{-}$event shape distributions, Nucl. Phys. B 407 (1993) 3 [InSPIRE].

[41] R.W.L. Jones et al., Theoretical uncertainties on $\alpha_{s}$ from event shape variables in $e^{+} e^{-}$ annihilations, JHEP 12 (2003) 007 [hep-ph/0312016] [INSPIRE]. 
[42] S. Schumann and F. Krauss, A parton shower algorithm based on Catani-Seymour dipole factorisation, JHEP 03 (2008) 038 [arXiv:0709.1027] [INSPIRE].

[43] S. Frixione and B.R. Webber, Matching NLO QCD computations and parton shower simulations, JHEP 06 (2002) 029 [hep-ph/0204244] [INSPIRE].

[44] S. Hoeche, F. Krauss, M. Schonherr and F. Siegert, A critical appraisal of $N L O+P S$ matching methods, JHEP 09 (2012) 049 [arXiv:1111.1220] [INSPIRE].

[45] S. Hoeche, F. Krauss, M. Schonherr and F. Siegert, QCD matrix elements + parton showers: the NLO case, JHEP 04 (2013) 027 [arXiv:1207.5030] [INSPIRE].

[46] F. Cascioli, P. Maierhofer and S. Pozzorini, Scattering amplitudes with open loops, Phys. Rev. Lett. 108 (2012) 111601 [arXiv:1111.5206] [INSPIRE].

[47] J.-C. Winter, F. Krauss and G. Soff, A modified cluster hadronization model, Eur. Phys. J. C 36 (2004) 381 [hep-ph/0311085] [INSPIRE].

[48] T. Sjöstrand, The Lund Monte Carlo for jet fragmentation, Comput. Phys. Commun. 27 (1982) 243 [INSPIRE].

[49] T. Sjöstrand, S. Mrenna and P.Z. Skands, PYTHIA 6.4 physics and manual, JHEP 05 (2006) 026 [hep-ph/0603175] [inSPIRE].

[50] A. Buckley et al., Rivet user manual, Comput. Phys. Commun. 184 (2013) 2803 [arXiv: 1003.0694] [INSPIRE].

[51] T. Gehrmann et al., NLO QCD matrix elements + parton showers in $e^{+} e^{-} \rightarrow$ hadrons, JHEP 01 (2013) 144 [arXiv:1207.5031] [INSPIRE].

[52] ALEPH collaboration, Studies of QCD at $e^{+} e^{-}$centre-of-mass energies between $91 \mathrm{GeV}$ and 209 GeV, Eur. Phys. J. C 35 (2004) 457 [inSPIRE].

[53] S. Höche, D. Reichelt and F. Siegert, Momentum conservation and unitarity in parton showers and NLL resummation, JHEP 01 (2018) 118 [arXiv:1711.03497] [INSPIRE].

[54] T. Sjöstrand, S. Mrenna and P.Z. Skands, A brief introduction to PYTHIA 8.1, Comput. Phys. Commun. 178 (2008) 852 [arXiv:0710.3820] [INSPIRE].

[55] M. Bahr et al., HERWIG++ physics and manual, Eur. Phys. J. C 58 (2008) 639 [arXiv:0803.0883] [INSPIRE].

[56] S. Höche and S. Prestel, The midpoint between dipole and parton showers, Eur. Phys. J. C 75 (2015) 461 [arXiv: 1506.05057] [INSPIRE].

[57] A. Verbytskyi et al., High precision determination of $\alpha_{s}$ from a global fit of jet rates, JHEP 08 (2019) 129 [arXiv: 1902.08158] [INSPIRE].

[58] A.H. Hoang, S. Mantry, A. Pathak and I.W. Stewart, Nonperturbative corrections to soft drop jet mass, arXiv:1906.11843 [INSPIRE].

[59] A. Buckley et al., General-purpose event generators for LHC physics, Phys. Rept. 504 (2011) 145 [arXiv: 1101.2599$]$ [INSPIRE].

[60] M. Dasgupta, L. Magnea and G.P. Salam, Non-perturbative QCD effects in jets at hadron colliders, JHEP 02 (2008) 055 [arXiv: 0712.3014] [INSPIRE].

[61] S. Marzani, L. Schunk and G. Soyez, The jet mass distribution after soft drop, Eur. Phys. J. C 78 (2018) 96 [arXiv:1712.05105] [INSPIRE]. 
[62] A. Banfi, H. McAslan, P.F. Monni and G. Zanderighi, A general method for the resummation of event-shape distributions in $e^{+} e^{-}$annihilation, JHEP 05 (2015) 102 [arXiv:1412.2126] [INSPIRE].

[63] S. Catani, B.R. Webber and G. Marchesini, QCD coherent branching and semiinclusive processes at large $x$, Nucl. Phys. B 349 (1991) 635 [INSPIRE].

[64] J. Bellm et al., HERWIG \%.0/HERWIG++ 3.0 release note, Eur. Phys. J. C 76 (2016) 196 [arXiv: 1512.01178] [INSPIRE].

[65] B.R. Webber, A QCD model for jet fragmentation including soft gluon interference, Nucl. Phys. B 238 (1984) 492 [INSPIRE].

[66] S. Gieseke, C. Rohr and A. Siodmok, Colour reconnections in HERWIG++, Eur. Phys. J. C 72 (2012) 2225 [arXiv:1206.0041] [INSPIRE].

[67] D. Reichelt, P. Richardson and A. Siodmok, Improving the simulation of quark and gluon jets with HERWIG 7, Eur. Phys. J. C 77 (2017) 876 [arXiv:1708.01491] [INSPIRE].

[68] R. Corke and T. Sjöstrand, Interleaved parton showers and tuning prospects, JHEP 03 (2011) 032 [arXiv: 1011.1759] [INSPIRE]. 